\title{
Acoustic emission description from a damage and failure scenario of rotomoulded polyolefin sandwich structure subjected to internal pressure for storage applications
}

\author{
Eric Lainé ${ }^{1, *}$, Jean-Claude Grandidier ${ }^{1}$, Maxime Cruz ${ }^{1}$, Anne-Laure Gorge ${ }^{1}$, Claire Bouvy ${ }^{2}$, and Geert Vaes ${ }^{2}$ \\ ${ }^{1}$ Institut Pprime, CNRS, ISAE-ENSMA, Université de Poitiers, 86962 Futuroscope, France \\ 2 Total Research \& Technology Feluy, Zone Industrielle Feluy, 7181 Seneffe, Belgique
}

Received: 21 December 2018 / Accepted: 29 October 2019

\begin{abstract}
The aim of this paper is to study the damage mechanisms in a sandwich polymer structure that contains three layers: two polyolefin skins and the foam core (skin-foam-skin). Specific tests on structure associated with the acoustic emission (AE) technique and tomographic observations (RX) are used to identify the damage. Initially, a conventional tensile test was performed to correlate the acoustic emission with the initiation of plasticity and damage to a polyethylene sample. The results obtained are close to those observed in other studies and it is possible to separate the signal from cavitation and propagation of necking. The technique is then employed to capture the rupture of a polymer skin on a multilayer rotomoulded structure (bottle). Tests were carried out on this bottle under internal water pressure. Three tests are performed with more or less early interruptions in order to identify the first damage and understand their evolution. Different quantities (average frequency, RA value, etc.) are observed in order to quantify and understand the perceived damage. With the $\mathrm{AE} / \mathrm{RX}$ correlation and mechanical behaviour, a scenario of structural damage is proposed.
\end{abstract}

Keywords: Polymer / multilayer structures / damage / structures testing / acoustic emission

\section{Introduction}

Sandwich materials are currently being used in an increasing number of industrial applications such as the marine, automotive, aerospace and aerospace industries. Sandwich materials or sandwich structures are generally composed of two thin skins and a thicker lightweight core. The face skins are generally metal, composite (Kevlar, carbon or fiberglass) or polymer, while the core may be honeycomb, cellular foam or balsa. Skins and cores are mechanically separable. The properties of these materials can therefore be modified by tuning the proportion of their constituents or the properties of the constituents themselves. These adjustments therefore make it possible to achieve the desired properties to meet well identified applications. This type of structure is more advantageous than a traditional monolayer structure (metallic, composite or polymer) because it is characterized by its lightness, insulation, hardness, resistance to fatigue, flexibility, high level of rigidity, high flexural strength, low surface density, etc... Thus, sandwich structures offer great potential to designers.

\footnotetext{
* e-mail: eric.laine@ensma.fr
}

Researchers of oil major Total have developed a new sandwich material consisting of a foamed polyethylene (PE) layer between two polyolefin skin layer. The structure is produced in a process that is called rotational moulding [1-3], a polymer conversion technology specifically designed to produce hollow plastics parts. The production of polymer foam involves the dispersion of gases within a polymer matrix, with the gas phase being generated from a physical or chemical blowing agent. A foamed structure in the rotational molding process requires the polymer matrix to form a continuous melting phase, minimizing the loss of blowing gas released into the atmosphere and reducing the negative effect of incoherent polymer melting on the morphology of the bubble [4].

This type of sandwich material (skin-foam-skin, Fig. 1a and b) dramatically increases the bending stiffness for the same weight. New technology opens the door to lightweight structures by using polyolefins. It is easy to imagine its interest particularly for the automotive [5], aeronautical, storage and transport industries. For example with this technology (rotomoulding) an electric vehicle was produced with a chassis having a skin-foam-skin structure [5], with skins in BIO-TP Seal ${ }^{\circledR}$. If the process is perfectly mastered today, building and designing structural parts require knowledge of the behaviour and resistance 


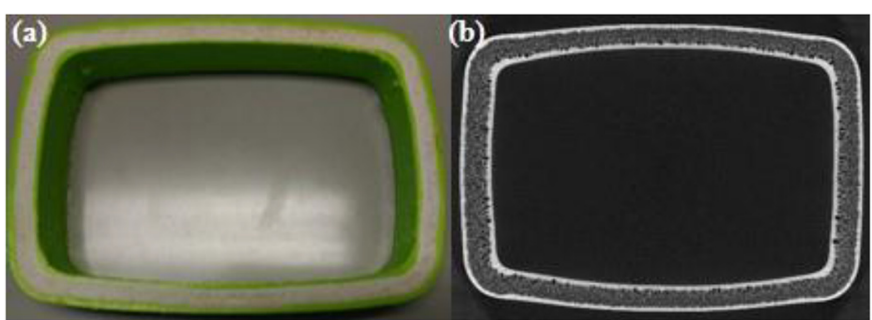

Fig. 1. (a) Photo of the section of a BIO-TP Seal ${ }^{\circledR}$ MultiLayer bottle. (b) Tomography of the section of a PE Multilayer bottle.

of such a material and more particular the evolution of damage under mechanical load.

The tests to characterize the mechanical behaviour of sandwich structures (or sandwich materials) in static and dynamic are generally bending (3-point or 4-point) and impact (weight drop) tests. In the literature, numerous works on these subjects and describing the different structures of materials are presented. Here are just a few of the many works describing the bending characterization [6-10] and falling weight impact test devices that have been reported [11-13]. In addition to the knowledge of the mechanical behaviour of these sandwich structures, the structural complexity of these ones requires additional means. Thus, to track the damage on these materials, a large number of authors mainly used the acoustic emission (AE) technique.

Acoustic emission has become an important nondestructive evaluation (NDE) technique in structures working under pressure by gas or liquid $[14,15]$. The AE technique for damage detection in carbon fibre composite pressure vessels has been evaluated for constant and cyclic internal gas pressure loading conditions [14]. AE was capable of monitoring the initiation and accumulation of damage events in a composite pressure vessel (CPVs), and it is possible to reliably distinguish carbon fibre breakage from other microscopic damage events (e.g. matrix cracks, fibre/matrix interfacial cracks). AE tests performed on the carbon fibre laminate used as the skin of pressure vessels revealed that the development of damage is highly variable under constant pressure, with large differences in the rupture life and acoustic emission events at final failure. Burman et al. [16] conducted the study of damage initiation and progression in cellular foam core sandwich composites using an acoustic emission. Acoustic characteristics of mode I and Mode II fracture of PVC foam were determined in Single Edge Notch Bend and End Notch Flexure tests. Core shear fracture initiation and growth were detected in four-point bending fatigue tests. Ben Ammar et al. [17] have investigated the mechanical behaviour under static and dynamic loadings and have evaluated damage by the acoustic emission method of two types of sandwich composite materials.

While some materials, due to their morphological structures or components (fibres), make it possible to capture a consequent acoustic activity (AA) during mechanical stress, the same is not true for thermoset and semi-crystalline polymeric materials. These materials are increasingly used in a wide range of applications (automotive, aerospace, transport...). However, the ex- treme conditions of use of these materials suggest that new methodologies are required to monitor deterioration of their properties in order to predict accurately their lifetime. Plastic deformation, damage and fracture mechanisms of semi-crystalline polymers have been extensively studied [18-22] and particularly for polyethylene [22-25]. Despite all this work, the characterization of the damage in $\mathrm{PE}$ remains a real challenge. The in situ determination of the initiation of microstructural plastic events or cavitation remains challenging and requires complex devices (SAXS, tomography, etc.). It was used to study these mechanisms in vitreous polymers [26,27], for example, Ronkay et al. [27] have recorded the acoustic activity emitted from PET during tensile tests. The authors attributed the signals to the formation of cavities, which appear with the neckpropagation. Nevertheless, Bohse [28] observed very low acoustic activity during plastic deformation of HDPE and PP in tensile tests. Qian et al. [29] recorded few AE signals during the deformation of a wide range of polymers including semi-crystalline polymers. While it is still difficult to conclude on the origin of $\mathrm{AE}$ signals, this technique has been used successfully by Galeski et al. [30] to collect $\mathrm{AE}$ signals during the spherulitic crystallization of semi-crystalline polymers from the melt. The authors attributed the release of acoustic waves to the formation of cavitation from melt. In their work, Teófilo et al. [31] analysed the rupture of poly (ethylene terephthalate) under tensile and relaxation conditions under stress and in contact with a sodium hydroxide solution. The use of acoustic emission, an unconventional technique, proved to be very useful for monitoring changes in the material during experiments. The authors were able to detect phenomena such as shear bands, the formation and location of cracks, and to assess polymer damage under different experimental conditions. Acoustic events related to stresses and deformations were determined for a number of situations, which is very useful in stress crack failure analysis. Recently, Casiez et al. [32] have shown for two types of PE samples that AE may be sufficiently sensitive to analyse the initiation of plastic strain, including cavitation, of semi-crystalline polymers above their glass transition temperature. Finally, Gomez et al.'s [33] study examined the use of $\mathrm{AE}$ techniques to monitor and characterize the effects of two different chemical agents (i.e., aqueous detergent and toluene) on the structure of a semi-crystalline polymer (high density polyethylene, HDPE) over different exposure periods. The characterization of the aged polymer using X-ray diffraction and tensile tests was correlated to the analysis of $\mathrm{AE}$ information based on signal and parameters.

The results presented in this literature review reinforce the use of $\mathrm{AE}$ assays as a promising technique for understanding structural changes in polymers. On laboratory specimens it is possible to connect the mechanisms (cavity - plastic strain) with the characteristics of the acoustic emission signals. On the other hand, very few studies used these results on the scale of polymer structures. For the intended application, these studies respond only very partially to the knowledge of the evolution of the mechanisms of damage in such a complex structure. 
The aim of the paper is to characterize the failure scenarios of a three-layer polymer structure, which does not exist in the literature, by combining RX and AE. However, one of the challenges is to verify whether this $\mathrm{AE}$ technique is capable of apprehending damage in a complex polymer structure of the type manufactured by Total (skin-foamskin). More precisely the representative structure used in this study is a multilayer bottle. The question to be addressed: is it possible with AE associated with tomography to obtain information on the damage in a three-layer structure. The difficulties with this system are: - the complexity of the sandwich structure (skin-foamskin),

- two different materials (PE foam, PE skins),

- different density materials,

- the internal environment (water),

- the "decimetric" dimensions of the bottle,

- the interface skin and foam which present a gradient of density.

In order to characterize the mechanisms with $\mathrm{AE}$ tools it is necessary to impose a particular protocol. The initial step is to identify the first acoustic signal associated with the first appearance of damage. Consequently, an analysis of the acoustic emissions during a tensile test can be carried out on polymer specimens which constitute the skin of the sandwich material and serve as a benchmark. At the scale of the structure different loading-unloading internal pressure should be applied on a multilayer bottle equipped with multiple sensors. A second challenge is to locate the area of the first damage in a complex multilayer structure. To link these signals to the damage, tomographs of the bottles in the initial state are compared with the scans made after each interruption of a test.

In the following paragraph, the material samples and structure, experimental systems and acoustic emission sensors are presented in detail in the experimental procedure paragraph. The results and discussions paragraph presents the results of the tensile test and the three specific protocols applied to the bottle before concluding. The tensile allows characterizing the parameters corresponding to initiation of damage (correlation). In the first experiment on the structure, the pressure is increased and the test is stopped when the first event appears. Loadingunloading steps with an increase of pressure are applied during the second test, the aim is to identify the first failure of a skin and its location. In order to identify the kinetics of damage a growing load is applied until breaking.

\section{Material and structure}

\subsection{Material}

The material is Lumicene ${ }^{\circledR} \mathrm{mPE}$ M4041 UV which is a new generation metallocene medium density polyethylene (mMDPE) with hexene as comonomer having a density of $0.940 \mathrm{~g} / \mathrm{cm}^{3}$. The structure is produced by rotational moulding, a polymer conversion technology specifically designed to produce the foam with a density of $0.200 \mathrm{~g} / \mathrm{cm}^{3}$.
A second material is obtained by combining polylactic acid (10\% PLA) with polyolefins (Lumicene ${ }^{\circledR}$ mPE M4041 UV). Developed by Total in collaboration with Persico, BIO-TP Seal ${ }^{\circledR}$ features innovative structural characteristics and ensures a superior glossy finish. Now components that require high surface shininess can be manufactured using rotomoulding. This last material has a rigidity about $20 \%$ higher than the Lumicene ${ }^{\circledR}$ mPE M4041 UV.

The tensile test specimens that are tested in this work are extracted from monolayer rotomoulded bottles with the Lumicene $^{\circledR}$ mPE M4041 UV material.

\subsection{Structure (bottle)}

A sandwich structure consists of a foam layer between two layers of polyolefin skin. The first two bottles tested are the sandwich structure consisting of a foamed polyethylene (PE) layer between two PE skin layers. The third bottle tested is the sandwich structure consisting of a foamed polyethylene (PE) layer between two BIO-TP Seal ${ }^{\circledR}$ skin layers. More precisely the dimensions of multilayer bottles are: height $330 \mathrm{~mm}$, width $200 \mathrm{~mm}$ and depth $140 \mathrm{~mm}$ (Fig. 2a-d). The section of the bottle is rectangular, the bottom is slightly curved and the edges have external and internal curvature radii of $15 \mathrm{~mm}$ and $5 \mathrm{~mm}$, respectively. For both materials, the skins and foam have a thickness of 2 and $10 \mathrm{~mm}$, respectively. A threaded tip is present at the top of the bottle. It is removed by machining here to install the jaws that maintain and seal the bottle during pressure testing (see next paragraph - Fig. 4).

Remark: this is a reference test structure for the company Total. It is used during the development of new materials to define and optimize the process parameters, to extract specimens for a thermo-mechanical characterization and to perform tests under different loading cases (creep, etc.).

\section{Experimental procedure}

\subsection{Tensile tests}

Uniaxial tensile tests were performed in an Instron Testing machine 1195 which is equipped with a $500 \mathrm{~N}$ load cell. Temperature was regulated at a constant value $\left( \pm 1^{\circ} \mathrm{C}\right)$. The tensile tests are carried out at one constant strain rate $1.75 \times 10^{-3} \mathrm{~s}^{-1}$ with the Videotraction system and performed at ambient temperature. Strains are computed from the displacement of four marks laid at the surface of the specimen using the Videotraction ${ }^{\circledR}$ software (distributed by ProViSys Engineering, France). During tests, the four marks are followed in real time by a CCD camera (resolution $800 \times 600$ pixels and frame rates $1.875-15 \mathrm{fps}$ ). The logarithmic longitudinal $\left(\varepsilon_{l}\right)$ and transverse $\left(\varepsilon_{t}\right)$ strains are calculated following equation:

$$
\begin{gathered}
\varepsilon_{l}=\ln \left(\frac{L}{L_{0}}\right)=\ln \left(1+\frac{\Delta L}{L_{0}}\right), \\
\varepsilon_{t}=\ln \left(\frac{W}{W_{0}}\right)=\ln \left(1+\frac{\Delta W}{W_{0}}\right),
\end{gathered}
$$



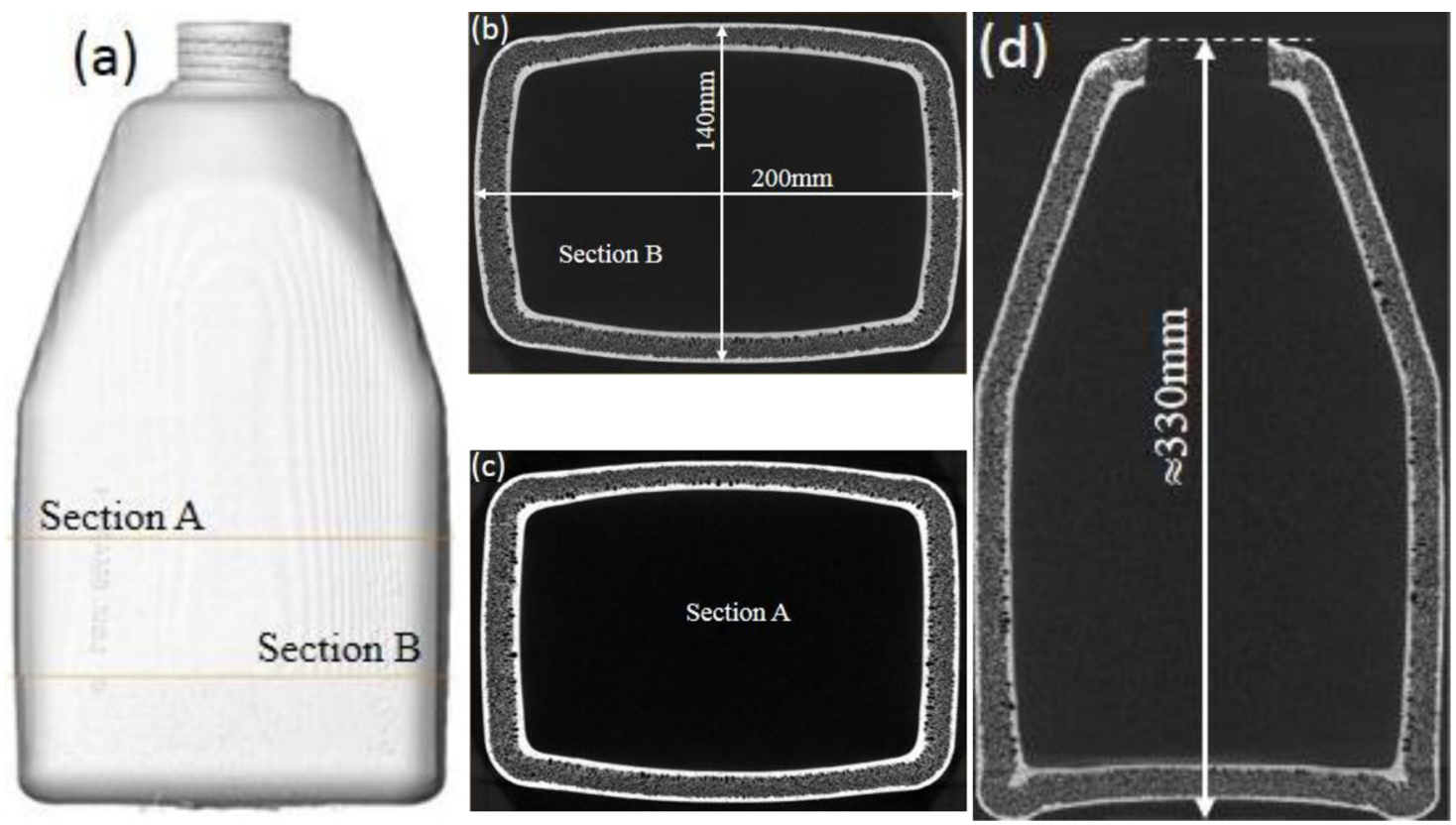

Fig. 2. (a) Bottle tomography. (b) and (c) Two transverse sections tomography. (d) Tomography of a longitudinal (bottle axis) section of the bottle.

where $L$ and $L_{0}, W$ and $W_{0}$ respectively stand for the current and initial distance between longitudinal and transverse (in width direction) marks, $\Delta L$ and $\Delta W$ the increments of these values.

The longitudinal Cauchy stress (or True stress) is calculated following (3), in isochoric framework and assuming isotropy of transverse strains (in width and thickness):

$$
\sigma_{v}=\frac{F}{S}=\frac{F}{S_{0}} \frac{1}{\exp \left(2 \varepsilon_{t}\right)}
$$

where $S_{0}$ is the initial cross-section area, $S$ is actual crosssection area and $F$ is the current axial load.

\subsection{Structure experimental methodology (ENDOMAT)}
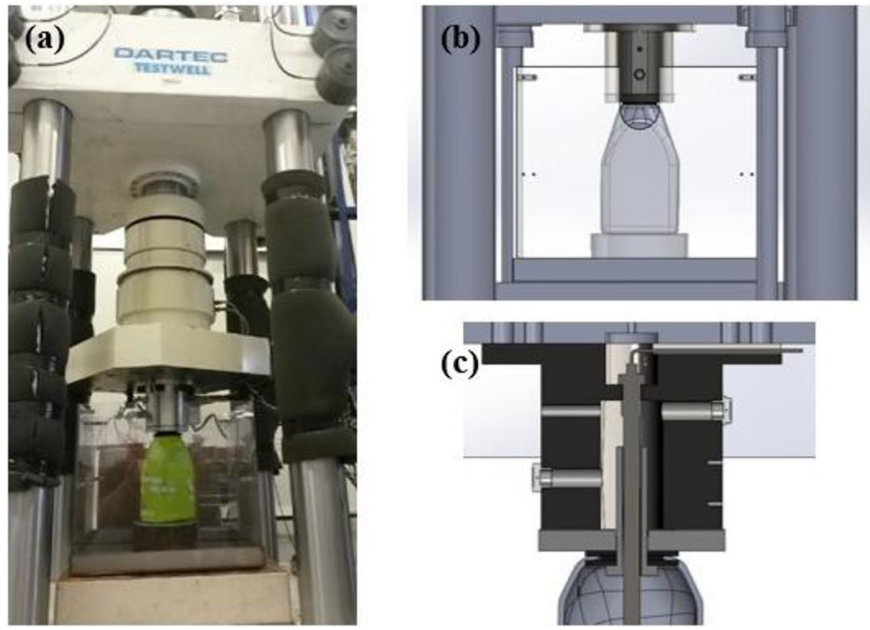

Fig. 3. (a) Positioning of the bottle on the test machine. (b) and (c) Specific assembly.

The experimental test bench Endomat (Fig. 3a) implements a mechanical testing machine tri-axial, including tensile-compressive, torsion and internal pressure. The limit values of the machine are $1200 \mathrm{kN}$ in tensilecompression, $55 \mathrm{kN}$ in torsion and 1200 bar in pressure test. The dimensions of the testing machine equal to $700 \times 700 \times$ $1800 \mathrm{~mm}$ with a volume of $882 \mathrm{~L}$ allow fastening largedimension structures on the bench test.

In this work, only pressure loading is used. The tension-compression axis is locked during all tests to position and maintain the bottle during testing. As of the pressure axis, it has two devices in parallel, each transforming the hydraulic power into oil to the hydraulic power circuit and into water in contact with the structure to be tested. The first is a transfer accumulator (oil or

water) that transfers the pressure supplied by the oil circuit with a storage tank (hydraulic operating pressure: 300 bar). The pressure transfer ratio is 1 but this system has the advantage of providing high flow rates (about the volume: $24 \mathrm{~L}$; rates of steering servo: $520 \mathrm{~L} / \mathrm{min}$ and $75 \mathrm{~L} / \mathrm{min}$ ) responding to problems of the large deformation of test structures. The second device is a booster that increases the pressure by four, thanks to the piston surfaces. The system can convert the oil operating pressure from 300 bar to 1200 bar in the water circuit, but at a lower rate (volume: $5 \mathrm{~L}$; rates of the steering servo: $20 \mathrm{~L} / \mathrm{min}$ ). The bench test is composed by recent devices electronic piloting DOLI, which require the monitoring of PID to control the individual axes in effort for each study conducted. 


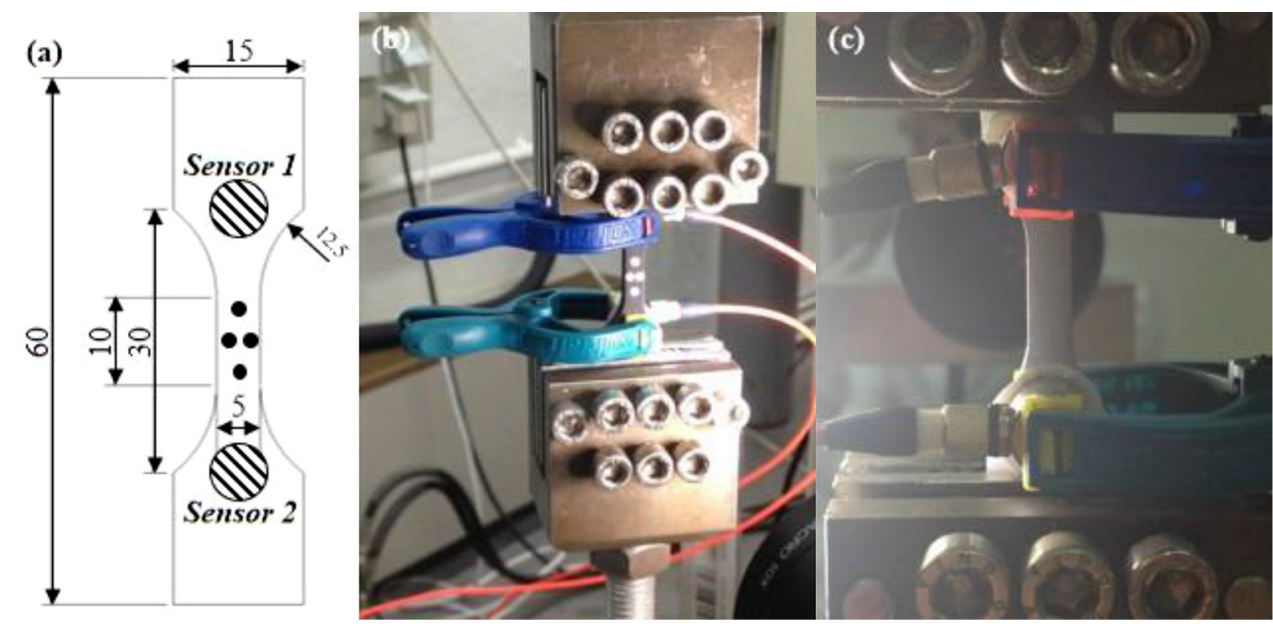

Fig. 4. (a) Schematic for the configuration of AE sensors and Markers on a tensile test specimen where dimension are given in millimeters. (b) and (c) Tensile test on PE specimen.

A specific assembly (Fig. 3b and c) has been developed to connect the bottle to the test bench, allowing positioning, maintenance and sealing during an internal pressure test. With this system, the bottle can be loaded with mechanical compression and internal pressure in different ways: monotonous, load-unload, creep, fatigue (cyclical). In addition, it is possible to couple mechanical compression and internal pressure.

\subsection{Acoustic emission}

\subsubsection{Acoustic emission for tensile test}

A two-channel PCI-2 from Mistras Group is used to record AE signals during tensile tests. The test is carried out with two "micro80" piezoelectric acoustic sensors with a PAC 1220 A preamplifier with a gain of $40 \mathrm{~dB}$. The acoustic emission signals are recorded by 2 piezoelectric Micro- 80 type differential sensors, $100 \mathrm{kHz}-1 \mathrm{MHz}$ bandwidth and $10 \mathrm{~mm}$ diameter, positioned on the tensile specimen with silicone grease and fixed by adhesive tape (Fig. 4). When recording AE signals, the user sets a detection threshold $(35 \mathrm{~dB})$ below which no signal is recorded. The amplitude distribution is $0-100 \mathrm{~dB}(0 \mathrm{~dB}$ corresponds to $1 \mu \mathrm{V}$ at the transducer output). The acquisition system is calibrated before each test using the mine breakage procedure [34,35]. This test makes it possible to estimate the propagation velocity and attenuation of acoustic waves in the material, which is $2000 \mathrm{~m} / \mathrm{s}$.

It should be noted that the elastic waves detected by the $\mathrm{AE}$ sensors and converted into a useful signal can also be called hit. The characteristics of a signal are well known such as duration, rise time and amplitude. Using two sensors, our setup enables linear location of AE signals along the gauge length of the specimen (Fig. 4a). The difference between the arrival times of a given wave at each sensor $\Delta t=\left(t_{1}-t_{2}\right)$ is calculated, and it is possible to determine the distance from the sensors of the $\mathrm{AE}$ signal assuming it occurred on the median line joining the centre of the two sensors, if mechanical damping is limited. Besides, only the signals whose intensity is big enough to reach both sensors can be localized and situated along the specimen. Hence, the signals are divided into two categories: the non-localized signals (so called "AE hits") and the localized signals (so called "AE events"). The position $P$ of those AE events is estimated according to the following relation:

$$
P=V\left(t_{1}-t_{2}\right)
$$

\subsubsection{Acoustic emission for structural tests}

An 8-channel Mistras Group 8-channel Express System is used to record AE signals during bottle testing. Damage monitoring is carried out using the acoustic emission measurement. All tests are carried out with eight piezoelectric acoustic sensors with a PAC 1220A preamplifier with a gain of $40 \mathrm{~dB}$ presented in previous paragraph. These are positioned on the bottle with silicone grease and fixed by adhesive tape (Fig. 5). When recording AE signals, the user sets a detection threshold $(32 \mathrm{~dB})$ below which no signal is recorded. In the same manner the propagation is evaluated by mine breakage procedure $[34,35]$. Timedependent parameters, such as amplitude, energy, duration, rise time, number of counts, etc., are calculated in real time by the acquisition system. Directly related to the shape of waveforms, these characteristics are widely used for AE analysis. In addition, we synchronized the acquisition of the internal pressure applied to the bottle (signal $0-10 \mathrm{~V}$ ).

The position of the sensors is in "square" (Fig. 5) on the two main sides. Special attention has been paid to the positioning and contact between the outer skin (PE or BIO-TP Seal ${ }^{\circledR}$ ) and the sensors by applying more force to them when inserting with silicone grease and tape strips. Acoustic response has been improved by this procedure. Thus, different location groups have been defined:

\footnotetext{
- a group for localization according to the 8 sensors: 3D localization (minimum 4 sensors),
} 


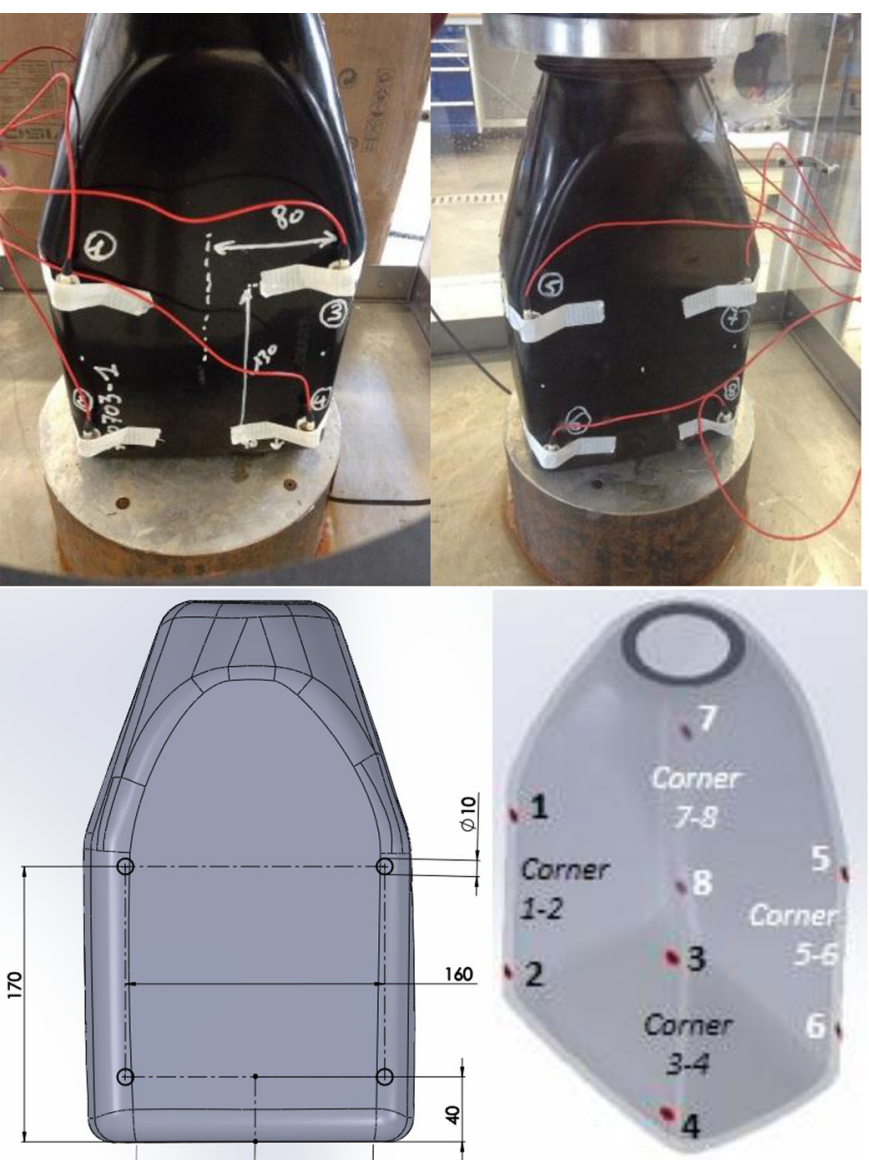

Fig. 5. Positioning of EA sensors (Test 1 and Test 2).

- two groups for each large face $(1,2,3,4)$ and $(5,6,7,8): 3 \mathrm{D}$ localization but according to a plan (minimum 3 sensors), - four groups for each edge $(1,2)(3,4)(5,6)(7,8)$ : linear localization (minimum 2 sensors),

- in order to possibly capture AE events during the tests.

\subsubsection{AE parameter analysis}

The first analysis that can be done during a test with acoustic emission is to represent the set of signals recorded in the amplitude - number of counts. This representation must be free of signals in the upper left and lower right corners in order to validate the system acquisition parameters. Signals with a small amplitude and a large number of counts correspond to mechanical noise, those with a large amplitude and a small number of counts correspond to electromagnetic noise [36].

On the other hand, AE parameter-based method (parameter analysis) has also been applied to crack classification based on the JCMS-III B5706 code [37] of which results were confirmed under the four-point bending tests and the direct shear tests of concrete specimens [38]. In order to classify active damage, AE parameters of the rise time noted $\tau_{r}$ (ms) and the maximum amplitude $A_{\mathrm{mv}}$ (Volts) are applied to calculate RA value (ms/V), and the average frequency noted A-FRQ $(\mathrm{kHz})$ is obtained from
$\mathrm{AE}$ count $\mathrm{AE}_{\mathrm{C}}$ and the duration time $\tau_{d}(\mathrm{~ms})$ as:

$$
\mathrm{RA}=\tau_{r} / A_{\mathrm{mv}},
$$

$$
\mathrm{A}-\mathrm{FRQ}=\mathrm{AE}_{C} / \tau_{d}
$$

The maximum amplitude $A_{\mathrm{mdB}}$ in decibels of an $\mathrm{AE}$ signal in time domain is given by the following formula:

$$
A_{\mathrm{mdB}}[\mathrm{dB}]=\left(20 \log A_{\mathrm{mv}} 10^{6}[\mu \mathrm{volt}]\right)-G,
$$

where the voltage in $\mu$ volt is $A_{\mathrm{mv}}$ and $G$ is the preamplifier gain. From these two parameters (RA value and the average frequency), it is possible to classify mechanisms manifesting themselves in different families of materials. For example, cracks are readily classified into tensile and shear cracks for concrete [38]. These parameters will be analysed for the different protocols. However, a defined criterion on the proportion of the RA value and the average frequency for crack classification has not been confirmed [38]. Finally, two other parameters can be analysed: the AE signal count $(N)$ and amplitude $\left(A_{\mathrm{mv}}\right)$. In general, the $\mathrm{AE}$ signal count $(N)$ and $A_{\mathrm{mv}}$ amplitude in $\mathrm{mV}$ show the following relationship $[39,40]$ :

$$
N=\alpha A_{\mathrm{mv}}{ }^{-m},
$$

where $\alpha$ and $m$ are constants [40]. In the equation $m$ is negative, implying the AE signals of large amplitude are observed less often than those of small amplitude. For fatigue crack growth of a steel material, it is reported that $m \approx 2$ [41]. Yamabe et al. [40] determined that $m=1.8$ from the rubber material for the static growth test. Thus, they conclude that since the value $m$ obtained from the static growth of rubber is consistent with that obtained from the growth of fatigue cracks in steel, this value is considered to be related to failure behaviour, i.e., crack growth [39].

In the case of polymers, damage modes are reduced, the mechanisms that can generate acoustic waves are viscoplasticity (high speed), cavitation - coalescence - tearing. At the scale of a complex structure, damping increases the difficulty and does not permit to analyse counts and events. In order to propose in this work a first classification for damage in a polymer structure, principally the classical $\mathrm{AE}$ descriptors are analysed as the RA value and the A-FRQ and the parameters of equation (8).

\section{Results and discussions}

\subsection{Evaluation on tensile specimen}

During the tensile test on the Lumicene ${ }^{\circledR} \mathrm{mPE}$ M4041 UV specimen, $24 \mathrm{AE}$ events and $357 \mathrm{AE}$ hits located in the area between the sensors are emitted and distinguished during acoustic emission recording. Figure 6a shows the number of counts as a function of amplitude. By observing the distribution, it appears that the measurements are reliable and are therefore not due to mechanical noise and/or electromagnetic noise. Figure $6 \mathrm{~b}$ first shows the time evolution of load and true stress on total duration of the tensile test. The amplitude of the received acoustic signals 


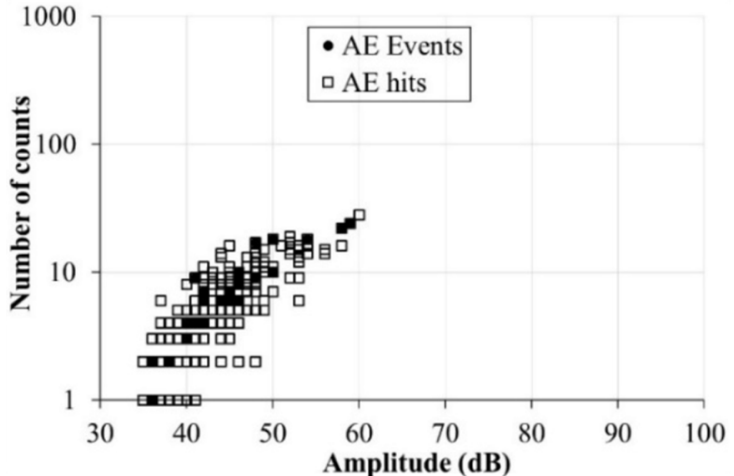

(a)

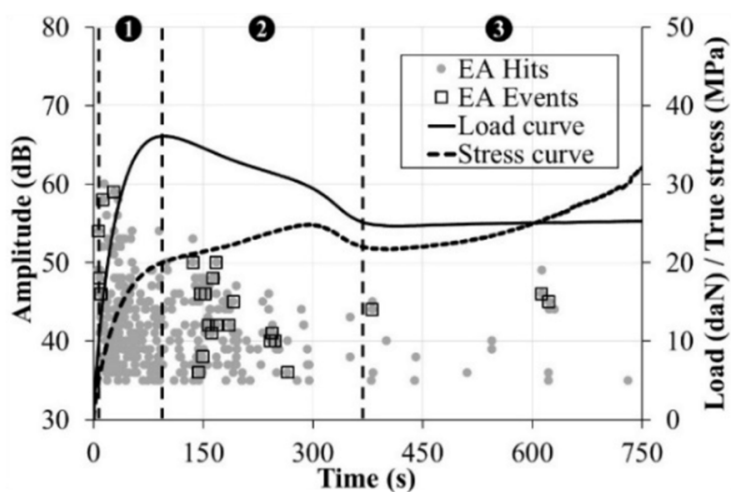

(b)

Fig. 6. (a) Number of counts vs amplitude. (b) Amplitude vs time, load and true stress vs time.

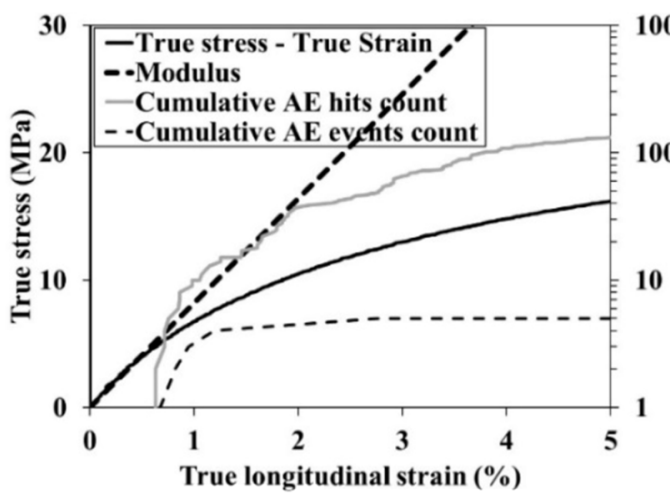

(a)

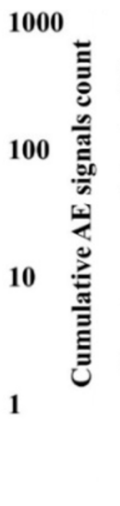

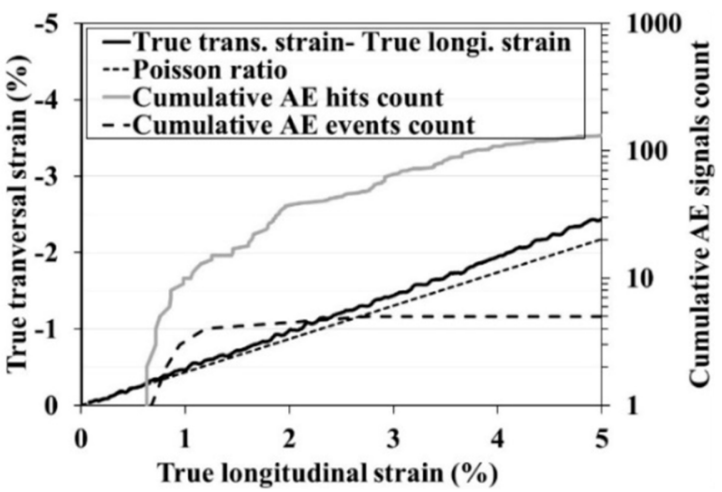

(b)

Fig. 7. (a) True stress and (b) true transversal strain and cumulative AE events and AE hits vs true longitudinal strain (5\% max).

is superimposed. In relation to the load curve three zones are distinguished: the first from the beginning of the test to the maximum of the load corresponding to first damage (cavitation) and the neck initiation, then the second zone corresponding to the decreasing force (neck location) and finally the third where we observe the load plateau corresponding to neck propagation [42]. The amplitude of the acoustic signals is between 35 and $60 \mathrm{~dB}$. There is a decrease in the mean amplitude during the test. The maximum is reached in the first zone, well before the neck initiation. When mechanical loading increases acoustic emissions are mainly generated between the first knee of the load curve and the point of maximum load. This corresponds to the development of cavitation in the useful zone. During the evolution of necking phase, activity AE strongly decreases and signals have lower amplitudes (35 at $40 \mathrm{~dB})$. The parameters of the signals are correlated with each other by plotting the amplitude according to the number of counts, this distribution is similar to [32], when the amplitude increases the number of counts increase. The acoustic data measured during this test are in perfect agreement with those measured by Casiez et al. [32].

For better correlation between the cumulative acoustic signals counts (AE events and $\mathrm{AE}$ hits) and the tensile properties, the true stress-true strain curve (Figs. 7a and 8a) and the true transverse strain-true longitudinal strain curve (Figs. $7 \mathrm{~b}$ and $8 \mathrm{~b}$ ) were plotted on the same graph by converting time into strain (the deformation speed being constant $0.175 \% / \mathrm{s}$ ). Figure $7 \mathrm{a}$ and $\mathrm{b}$ are a zoom (the first 5 percent of strain) of the curves shown in Figure $8 a$ and b, respectively. For both curves (Fig. 7a and b), the slope at the origin makes it possible to capture the loss of non-linearity (straight in dashed line). Thus, for true stress-true strain and the true transverse strain-true longitudinal strain curves, the original slopes represent the elastic mechanical parameters of the material which are, respectively, the modulus and the Poisson's ratio. It appears that the first acoustic signals (AE hits and AE events) appear at about $\approx 0.6 \%$ true longitudinal strain and a stress of $\approx 5.5 \mathrm{MPa}$. No AE signal is detected during the linear phase of load curve. More precisely, as soon as the linearity between true stress and true strain, and, true transverse train and true longitudinal strain is lost, the first AE hits (and AE events) appear. There are $5 \mathrm{AE}$ events at the beginning of the true stress-true strain curve (Fig. 8a), with almost the highest amplitudes and the rest after the necking with lower amplitudes. From about 25\% strain, which corresponds to zone 2 where the force decreases (neck localization), the number of AE signals decreases strongly. This is seen in Figure $8 \mathrm{a}$ and $\mathrm{b}$ where the curves 


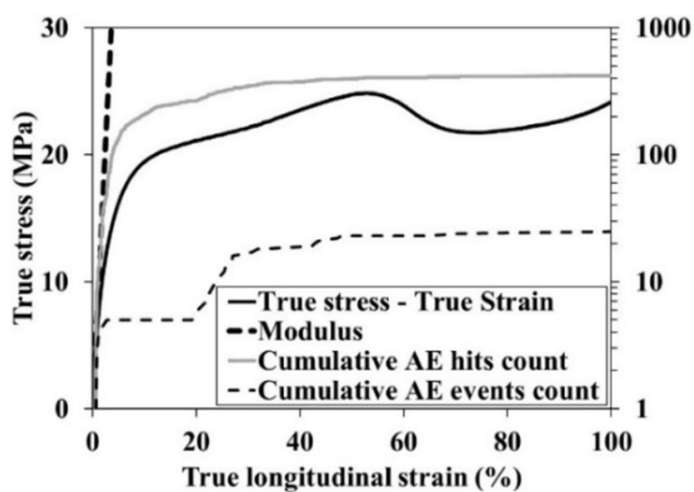

(a)

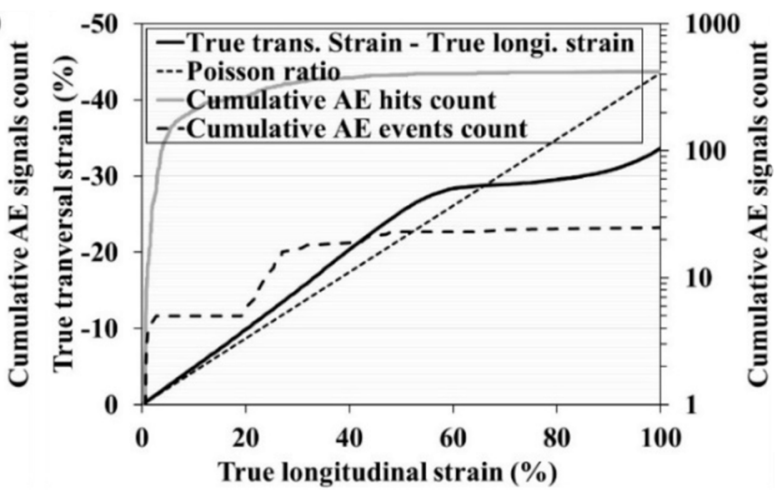

(b)

Fig. 8. (a) True stress and (b) true transversal strain and cumulative AE events and AE hits vs true longitudinal strain (100\%).

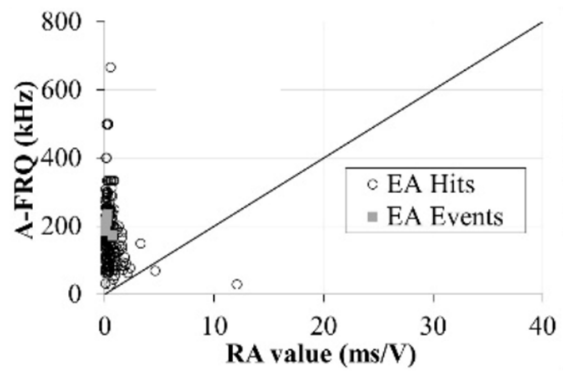

(a)

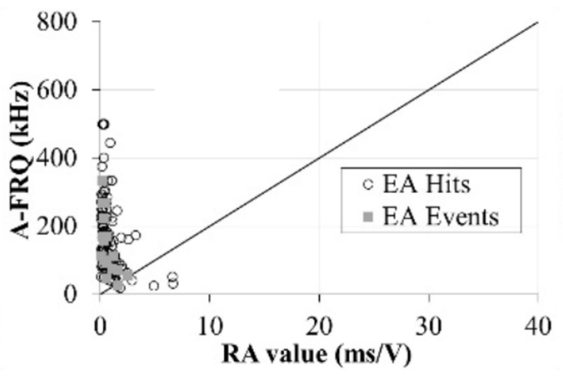

(b)

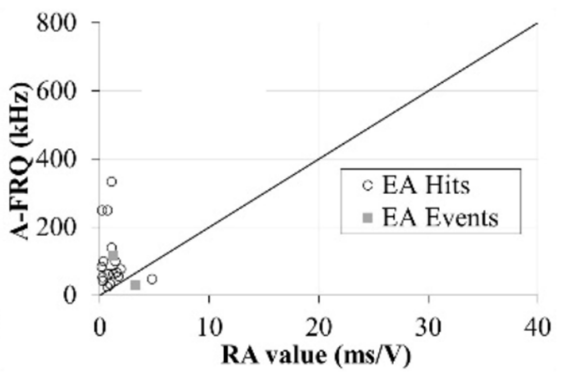

(c)

Fig. 9. Tensile specimen - average frequency vs RA value. (a) Zone 1 (Fig. 6b). (b) Zone 2 (Fig. 6b). (c) Zone 3 (Fig. 6b).

representing the cumulative number count of AE hits and AE events, respectively, tend towards a plateau.

Figure $9 \mathrm{a}-\mathrm{c}$ shows A-FRQ as a function of the RA value of each acoustic signal for zones 1, 2 and 3 defined in Figure $6 \mathrm{~b}$, respectively (the limits of the axes of this graph are set in order to discriminate the damage and to be able to compare easily with the results on bottles later on). These figures show that for a tensile test, the vast majority of $\mathrm{AE}$ signals have a high A-FRQ value and a low RA value. Unlike concrete, no studies to our knowledge have tried to use these parameters to classify damage. Obviously, a single test is not enough, especially a tensile test. However, it can be observed two sets: AE with high amplitude and high A-FRQ (RA value low) and those with low amplitude and High A-FRQ.

For other materials (concrete for example) tested under different loads, work in the literature showed that it was possible to distinguish two damage modes by a line separating the high A-FRQ and low RA value and the low A-FRQ and high RA value. This line is naturally material dependent. However, no work to date has given any indication on the slope of this line for a polymer. We arbitrarily choose the case that the proportion of the RA value and the average frequency is set to 1:20 as shown in Figure $9 \mathrm{a}-\mathrm{c}$. The majority of the signals are above this straight line, the few signals below correspond to the events at the end of loading when the necking unfolds with large values of longitudinal strains. Figure 10a and b shows the evolution of RA value and A-FRQ as a function of time with the superposition of the load and the three zones of evolution of the damage respectively. In Figure 10a and b, the dots correspond respectively to the RA value and to the A-FRQ of each AE hit while the solid line is the moving average of the recent 20 hits in order to show the trend clearly. The moving average line of the RA value in Figure 10a shows an increase in the RA value from 0.4 to $1.1 \mathrm{~ms} / \mathrm{V}$ in zones 1 and 2 and a smaller increase from 1.1 to $1.25 \mathrm{~ms} / \mathrm{V}$ in zone 3 . The moving average line of the A-FRQ in Figure 10b shows an initial value close to $200 \mathrm{kHz}$. In the first zone, this line decreases to $150 \mathrm{kHz}$ and then increases with a peak at $250 \mathrm{kHz}$ at the beginning of the striction (neck initiation). In zone 2 this value oscillates between 150 and $250 \mathrm{kHz}$, then decreases constantly in zone 3 to reach an average value of $100 \mathrm{kHz}$.

Figure 11a shows the number of AE signal counts for each amplitude in $\mathrm{mV}$. For amplitudes between 35 and $42 \mathrm{~dB}$ (corresponding to 5.6 and $12.6 \mathrm{mV}$ respectively in the Fig. 11a), the number of AE signal counts are close and it is possible to pass through these points a horizontal line (Fig. 11a). On the other hand, above $42 \mathrm{~dB}$ (corresponding to $12.6 \mathrm{mV}$ ), the distribution of the signals is such that the number of signals with a given amplitude decreases proportionally with the increase in their amplitude (Tab. 1, Fig. 11a). This evolution can be represented by 


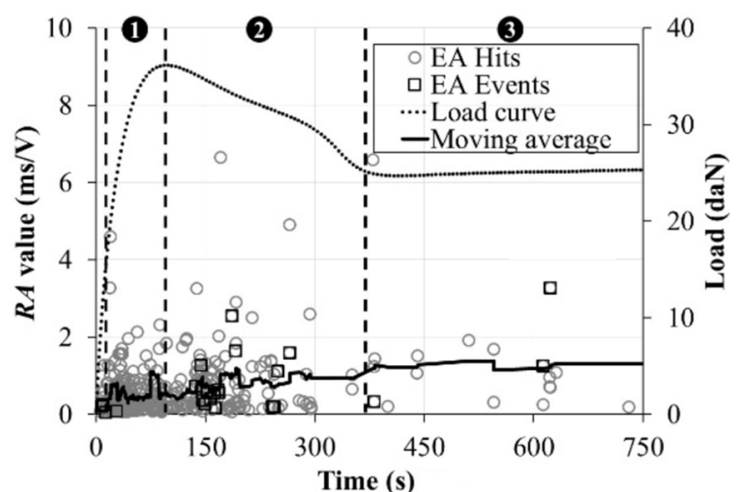

(a)

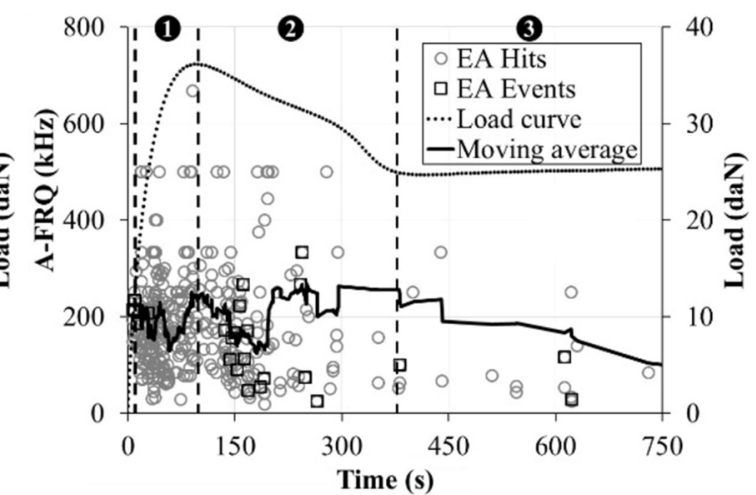

(b)

Fig. 10. Tensile specimen. (a) RA value and load vs time. (b) Average frequency and load vs time.

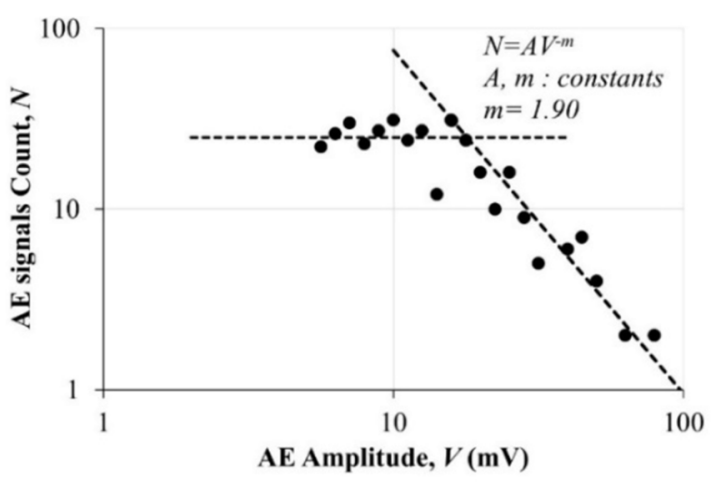

(a)

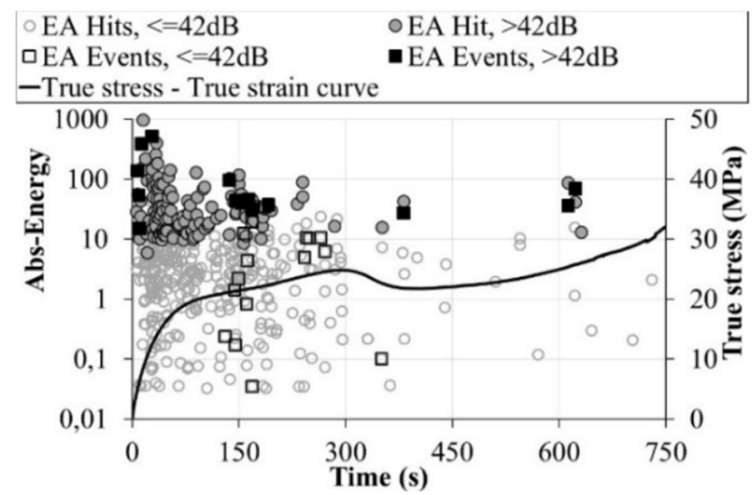

(b)

Fig. 11. (a) Relationship between AE signals count and AE amplitude obtained from static tensile test. (b) Abs energy and true stress vs time.

Table 1. Distribution of the AE signals according to the amplitude level in the three areas of the tensile test.

\begin{tabular}{|c|c|c|c|c|c|}
\hline & & \multicolumn{2}{|c|}{ AE events } & \multicolumn{2}{|c|}{ AE hits } \\
\hline & & $35-42 \mathrm{~dB}$ & $>42 \mathrm{~dB}$ & $35-42 \mathrm{~dB}$ & $>42 \mathrm{~dB}$ \\
\hline Zone 1 & First damage (cavitation) & 0 & 5 & 107 & 97 \\
\hline Zone 2 & Neck location & 10 & 7 & 91 & 43 \\
\hline Zone 3 & Neck propagation & 0 & 2 & 12 & 7 \\
\hline Total & & 10 & 14 & 210 & 147 \\
\hline Total & & \multicolumn{2}{|c|}{24} & \multicolumn{2}{|c|}{357} \\
\hline
\end{tabular}

equation (8) with a value $m$ of 1.90 . The amplitudes less than $42 \mathrm{~dB}$, a plateau appears in the AE signals count - AE amplitude plane. With regard to the tensile test, the recordings were not performed until the specimen failed but only at $100 \%$ strain. Thus, only the initiation of damage (high amplitudes in zone 1) and its coalescence tearing in zones 2 and $3(<42 \mathrm{~dB})$ are observed. It is very likely that if the test had been performed to failure, the tear would have generated additional signals in the $35-42 \mathrm{~dB}$ range. This is why we observe a plate that does not correspond to a mechanism but the consequence of a test stopped at $100 \%$ strain.

\subsection{Characterization of first damage on a sandwich structure (bottle)}

The objective of this first test on structure is to try to identify the relationship between the very first acoustic signals and the local damage in the bottle under increasing internal pressure. In order to determine this relationship, the test is interrupted as soon as the first acoustic signals are received. At each interruption, the bottle is disassembled, emptied and scanned in order to localize the first damage and then reassembled on the test bench to continue the test. One of the major difficulties of this procedure is to 


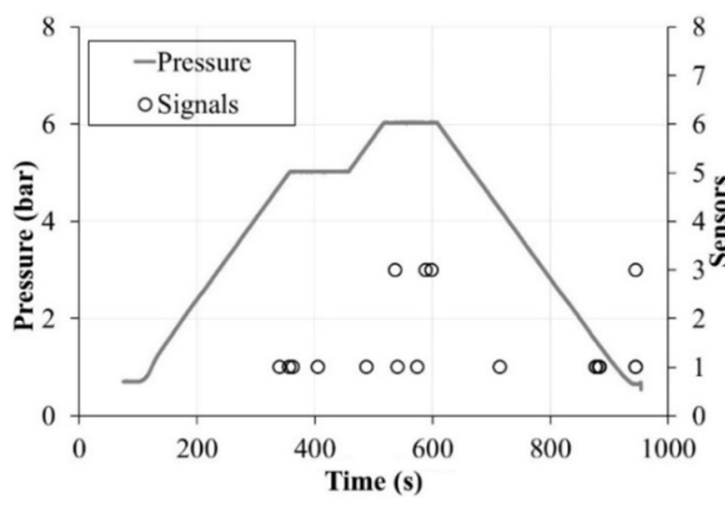

(a)

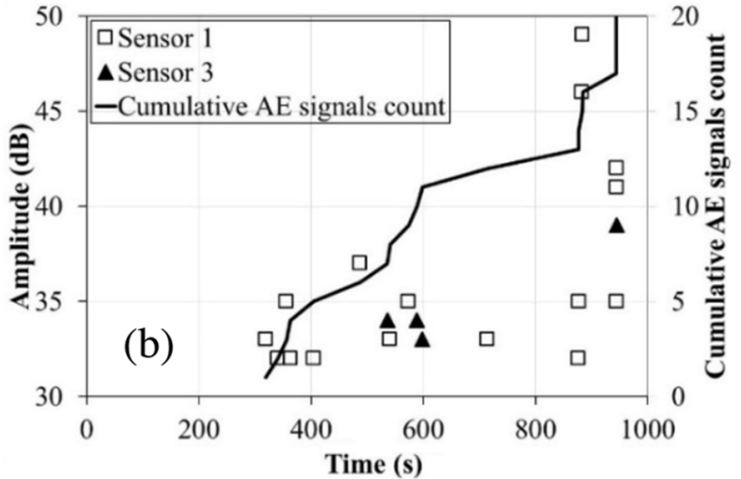

(b)

Fig. 12. Test 1. (a) Pressure and sensors vs time. (b) Amplitude and cumulative AE signals count vs time.

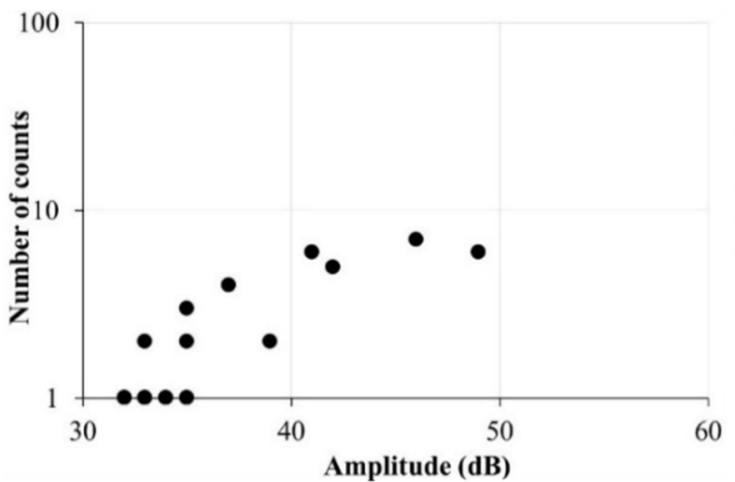

(a)

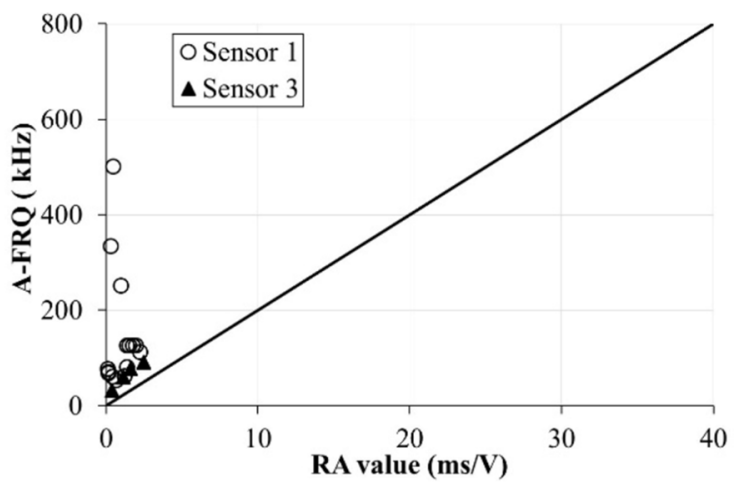

(b)

Fig. 13. Test 1. (a) Number of counts vs amplitude (dB). (b) Relationship between average frequency and RA value.

reposition perfectly between each test bottle and especially the acoustic sensors.

At the first loading, acoustic signals (only AE hits) are received on sensors $1,2,5$ and 7 . The maximum pressure is 2 bar. The exploitation of tomography does not provide any information to establish a link between the first AE hits and local degradation of the inner or outer skin. A second charging is carried out up to 5 bar and stopped when two acoustic signals appear on the monitor. Similar to the previous tomography it does not provide any information. The third test is interrupted very quickly because an AE signal appears at 1 bar. But once again, nothing is observed visually or by imagery. These first hits are either due to very local damage or to noise from the machine. However, the characteristics of the signals are very close to those observed at the point of loss of linearity in the traction curve. The signals have a very low RA value and a high A-FRQ. Of course, it is not possible to completely exclude machine noises but the comparison with the first events under traction is convincing enough to associate these events to the initiation of the first damage in the same manner as the tensile test. In this case, the tomography is not precise enough to locate and observe the consequences of these mechanisms which should be cavitation mechanism.
For the fourth load, it is envisaged to go up to 5 bar, and to continue by steps of 1 bar according to the detection rate of acoustic signals (Fig. 12a). An acoustic activity (AA) already appears between 4 and 5 bar on sensor 1 and then on sensor 3 between 5 and 6 bar (Fig. 12a). The test continues with a constant bearing at 6 bar. In addition to sensor 1 , acoustic signals are received by sensor 3 . The test is interrupted after these $10-11$ hits to scan the bottle again because as already stated, the aim is to correlate the AA and damage, and thus avoid an excessive breakage of a skin. At the end of the discharge, 2 new hits can be seen on sensor 1 with a slightly higher amplitude. Figure $12 \mathrm{~b}$ shows that during pressure relief, the acoustic activity accelerates slightly (9 hits between 400 and $1000 \mathrm{~s}$ ) with a higher intensity because the signal exceed $40 \mathrm{~dB}$. Note that the other sensors $(2,4,5,6,7,8)$ did not perceive any acoustic signals during the test. This first test suggests that the first hits are either the signature of the initiation of the first damage or perhaps parasitic machine and assembly events that are recorded with the required filtering (Fig. 13a). The first hits at $40 \mathrm{~dB}$ are well in tune with wall breaking mechanisms, which are the first mechanisms that appear during pressure build-up. The distribution of hits in Figure 13a are similar to those measured under tensile stress. The A-FRQ and RA characteristics match to 


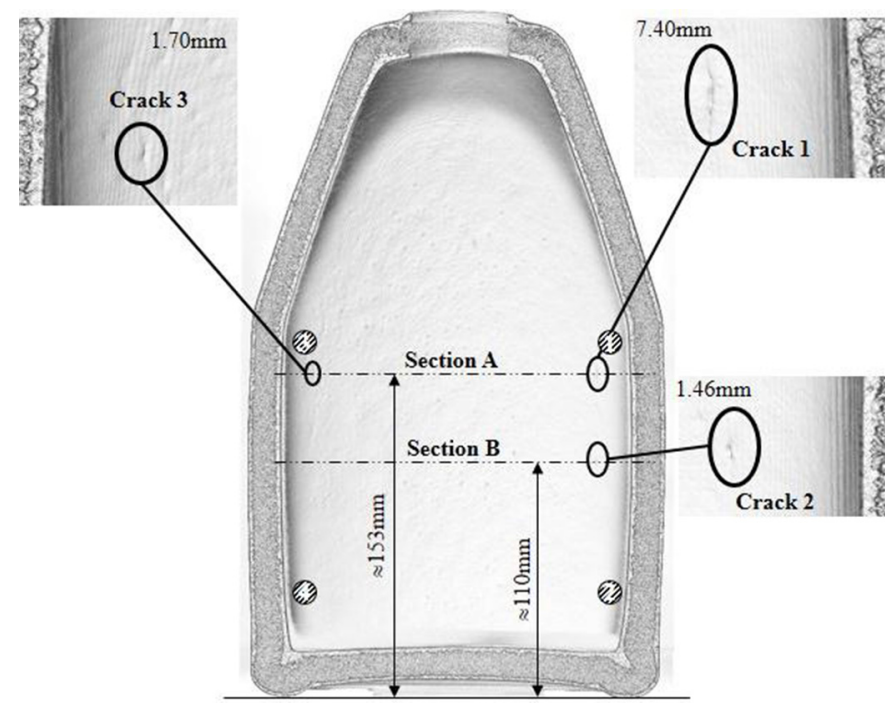

Fig. 14. Test 1. Bottle - location of rupture zone.

distribution of initiation of damage under the traction tests. It seems reasonable to associate these hits to initiation of damage and the first small crack in inner skin.

An analysis of acoustic emission suggests that they are located between the sensor pairs 1-2 and 3-4, and very close to sensors 1 and 3 . The tomography images of this area show that the inner skin of the bottle is beginning to damage itself (Figs. 14 and 15). A small crack has appeared in an area where the skin thickness is very thin, next to initial cavities at the skin/foam interface (Fig. 14). It is important to note that not all thinner areas are subject to this level of load damage. The first damage appeared near the bottom curvature, it is a more complex stress area than the bottle walls. No local breakage is observed on the opposite side (sensors from 5 to 8 ). The 20 hits captured during this test are distributed on the two sensors 1 and 3 , respectively, 16 and 4 hits. Locally measured cracks are $7.4 \mathrm{~mm}$ for crack 1 and $1.7 \mathrm{~mm}$ for crack 3 . It is very likely that crack 2 between sensors 1 and 3 is not captured. If a link can be made between the number of hits and the length of a crack, then we see that here the ratios are very similar, so this ratio is 0.463 for crack 1 and 0.425 for crack 3 . Figure 13b shows that the signature of these hits are very close to those observed in the damage phase of the specimen in traction. All points are above the slope line 20 .

\subsection{Identification of failure of a skin (bottle)}

In order to identify the critical load in relation to the failure of the inner skin, it is necessary to apply pressure (loaddischarge) to the new bottle in steps of 1 bar from 4 bar onwards. Figure 16a shows that the test and measurements of acoustic signals are not a priori disturbed by external noise (mechanical and electromagnetic). Figure 16b shows that the first hits appear at the end of the fourth load, between 6 and 7 bars, just before the tray. The four sensors $(1,3,5,7)$ placed at the top of the bottle (Fig. 5) and only sensors 2 and 4 placed at the bottom of the bottle received acoustic signals. The signals are mainly between sensor

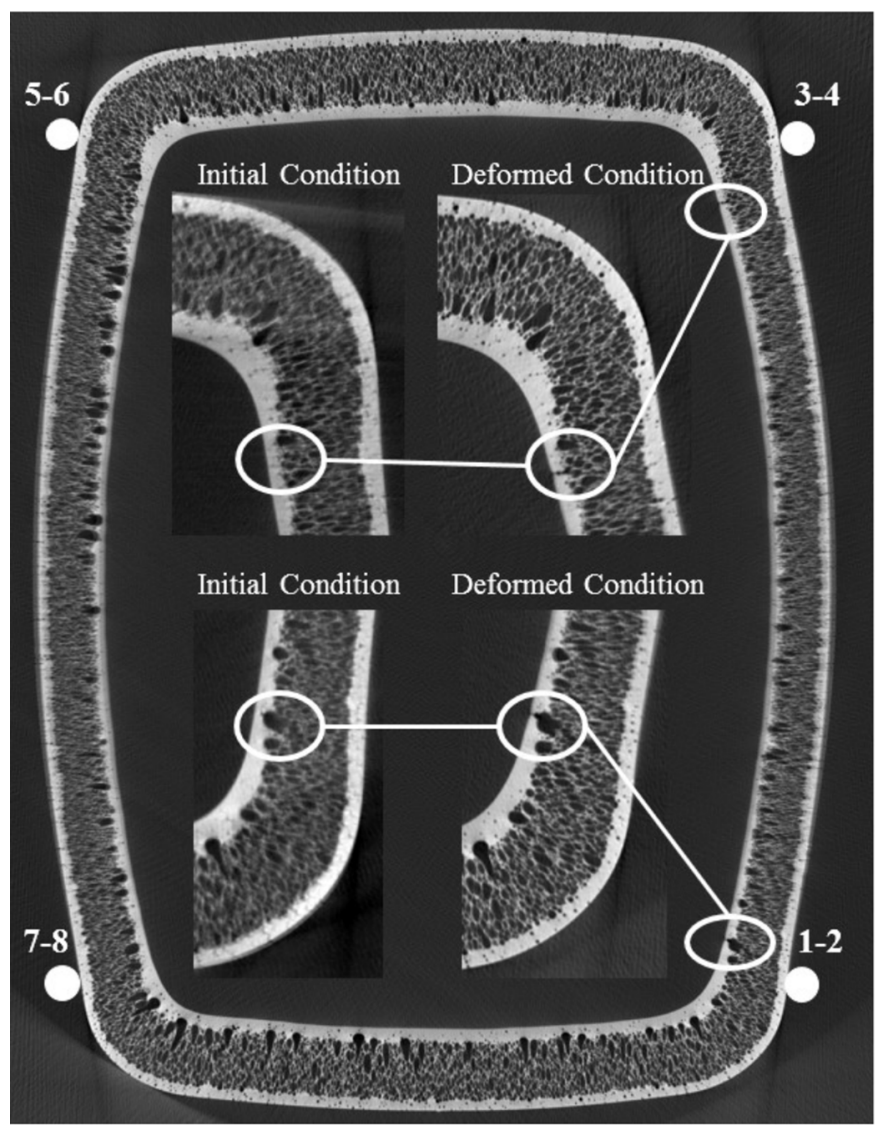

Fig. 15. Test 1. Section A of Figure 14-location of rupture zone.

pairs 1-2 and 3-4 (Fig. 17). Lower activity was observed on corners 5-6 and 7-8 and sensors 6 and 8 did not detect acoustic signals. Either they were incorrectly positioned or the intensity was too low to be detectable in these areas due to high damping of polymer. However, Figure 18 shows that the cracks at angles 5-6 and 7-8 are the smallest, respectively 55 and $60 \mathrm{~mm}$. Moreover, the distance between their ends and sensors 6 and 8 is still high, which does not allow acoustic signals to be detected. However, sensors 2 and 4 picked up signals and their quantities are proportional to the length of the measured cracks and especially to the position of their end. Indeed, the crack between sensors 3 and 4 is the longest, and sensor 4 is the one that detected the most hits. Finally, note that the amplitudes remain low since all the hits are below $45 \mathrm{~dB}$ with $93 \%$ of the population between 32 and $40 \mathrm{~dB}$.

After the fourth cycle a scan of tomography has been performed. In Figure 18, it can be seen that the inner skin actually shows multiple cracks on the height of the bottle, which are located on the face that has expanded the most and close to the corners with different lengths $(27 \mathrm{~mm}+$ $28 \mathrm{~mm}$ between sensors 5 and $6,86 \mathrm{~mm}$ between sensors 1 and $2,115 \mathrm{~mm}$ between sensors 3 and 4 ). Following the analysis of the tomography (Fig. 19), in the positioning section of the four sensors placed (section B in Fig. 19) at the top of the bottle, there are four luminous zones in the corners of the bottle, but on the side of the large face (those which inflate during the loading under pressure). 


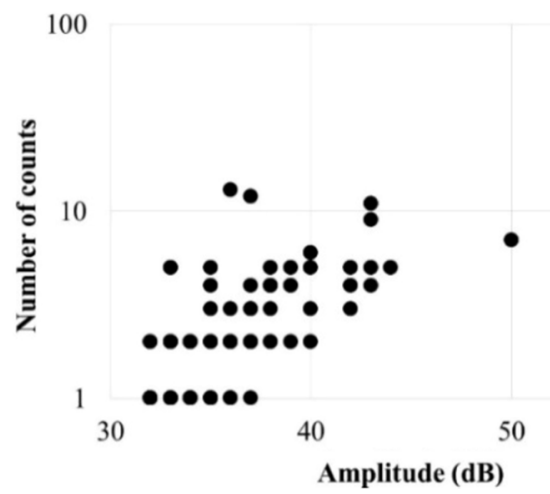

(a)

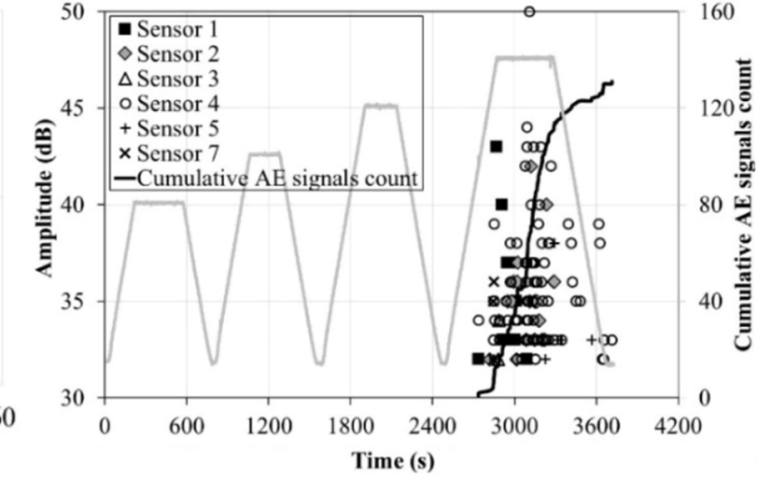

(b)

Fig. 16. Test 2. (a) Number of counts vs amplitude (dB). (b) Pressure load-unload in 1bar steps vs time - Amplitude of signals on different sensors vs time - cumulative AE signals count vs time.
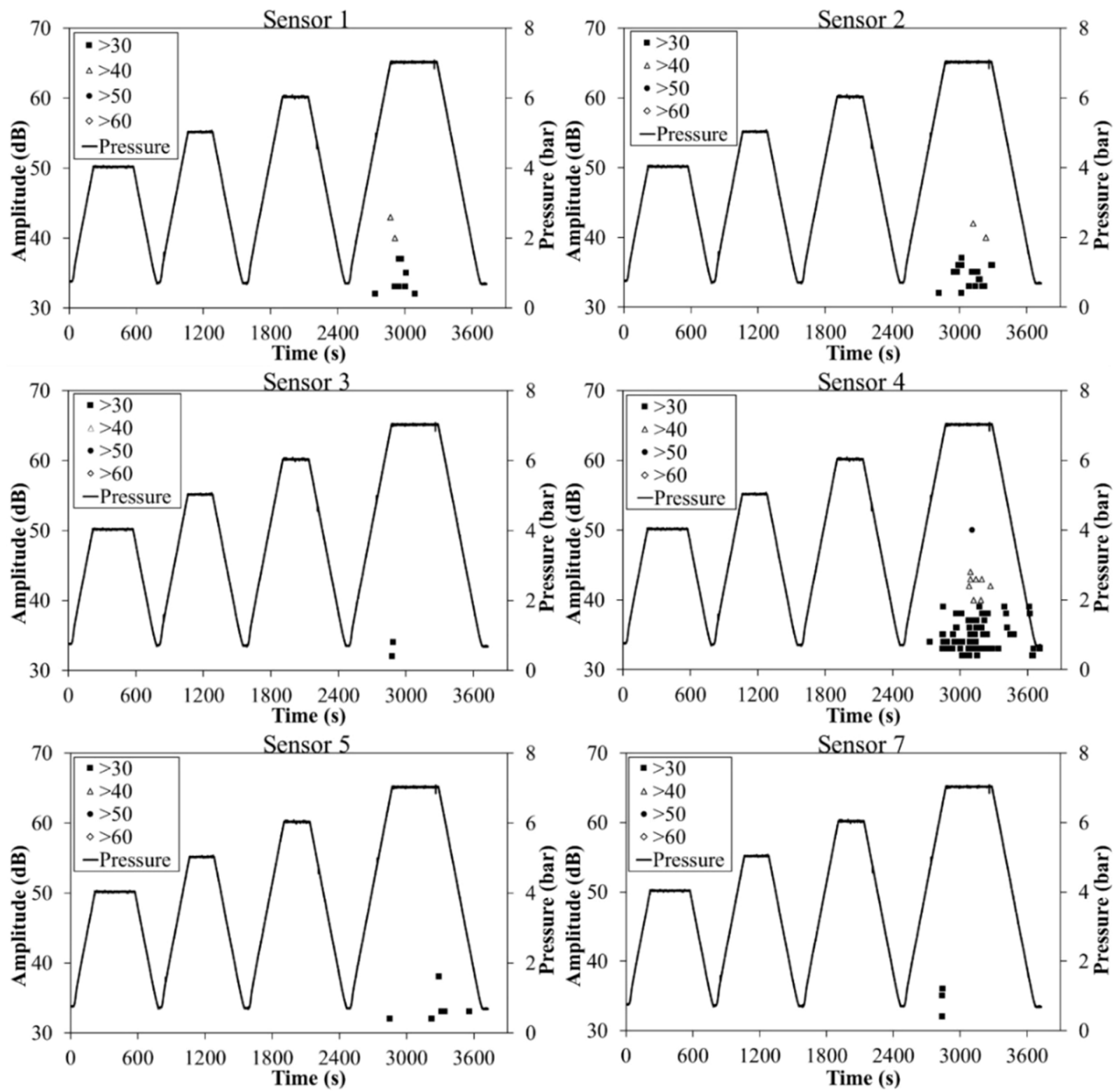

Fig. 17. Amplitude of signals on different sensors. 

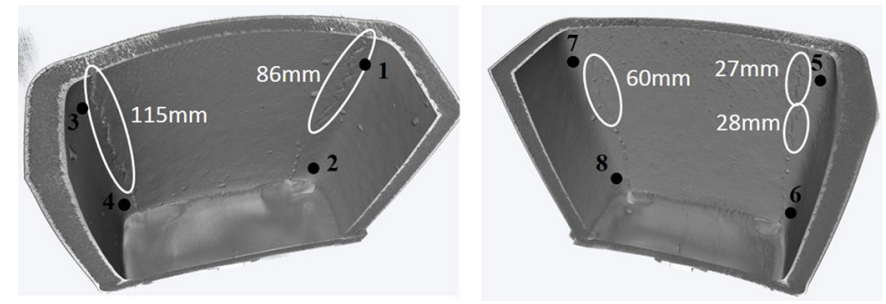

Fig. 18. Tomographs of the bottle after test 2 .

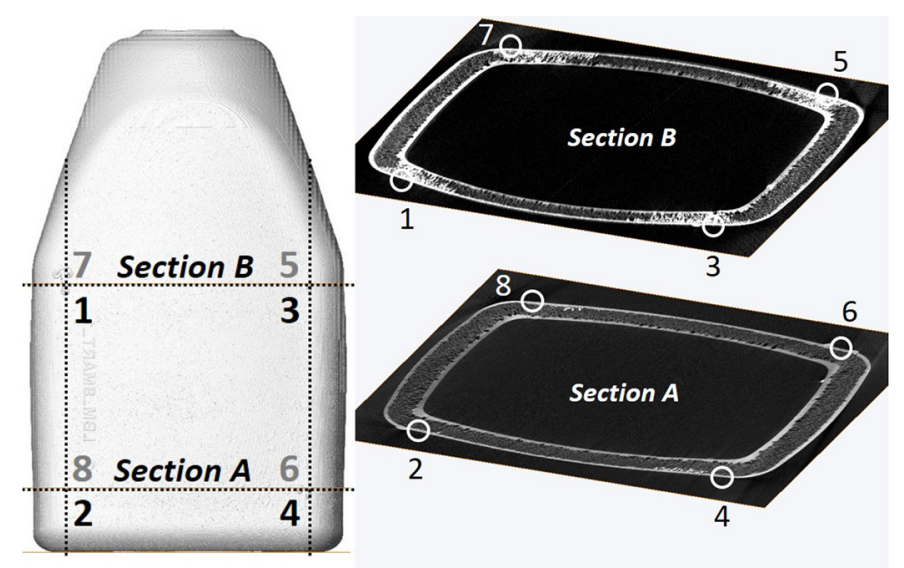

Fig. 19. Tomography of bottle section after test 2 .
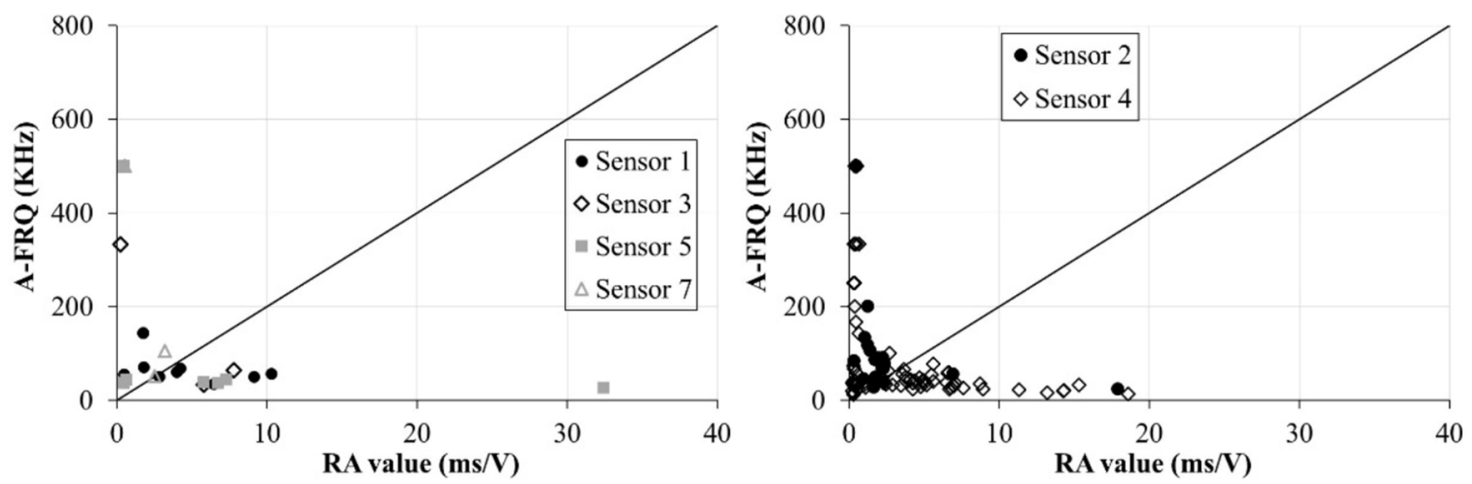

Fig. 20. Test 2 relationship between average frequency and RA value for each sensors.

This corresponds to water infiltration at the foam level, which confirms that the internal skin is locally damaged.

However, in Figure 18, where the bottle is cut approximately $25 \mathrm{~mm}$ above the sensor positioning plane, it appears that only the cracks located in corners 1-2 and 3-4 and to a lesser extent in corner 5-6 have propagated since highlighted areas are present. However, they are less widespread than the areas located in section B. Finally, at the level of the positioning plane of the other sensors $(2,4$, $6,8)$, section A on Figure 19, no sign indicates the presence of water at the level of the foam. Section B (Fig. 19) of the bottler is the near area where there is the most swelling due to internal pressure. Thus, the crack opens and thus water can penetrate into the foam part.

Compared to the previous test, the analysis of the acoustic emission signals shows that low A-FRQ and strong RA value hits appear, which correspond to the characteristics of the events observed under large strains in a tensile test. The tomography showed a tearing of the inner skin, unobservable in the previous test, consequently it is reasonable to associate these new hits to the spread of damage. This propagation involves localization of deformations and tearing. In Figure 20, as many hits with low and high RA values are generated which is a link to the process area with cavitation followed by propagation (tearing).

\subsection{Generalisation to other polyolefin}

A monotonous load test under internal pressure was performed on the BIO-TP Seal ${ }^{\circledR}$ bottle. Finally, for this last test, the positions of the sensors are modified in order to improve the location of the information on the bottle by AE. This change allows comparing the capabilities of two different positioning of sensors. Thus, the sensors are positioned in a "triangle" (Fig. 21) to locate the acoustic signals as accurately as possible because the localization system works by calculating the time difference of the acquisition of a wave for two different sensors. If the system is symmetrical, localization is even more difficult. After configuration and adjustment of the device, event location is possible on the outer skin of the bottle. Finally, the sensors are positioned as close as possible to the rupture zones detected by previous tests in order to optimize the reception of the acoustic sensor signal.

Thus, from 6 bar pressure (Fig. 22b), the AE signals intensify rapidly (Fig. 22b) and mainly on sensors 1, 2 and 3 (Fig. 23), but no activity of sensors 4, 5, 7 and 8 and a very low activity of 6 is observed. After disassembly of the entire assembly (bottle and specific assembly) of the machine, then after removing the specific assembly and completely emptying the bottle, it is visible that the inner skin of the bottle has several important cracks (Fig. 24). Thus, the 

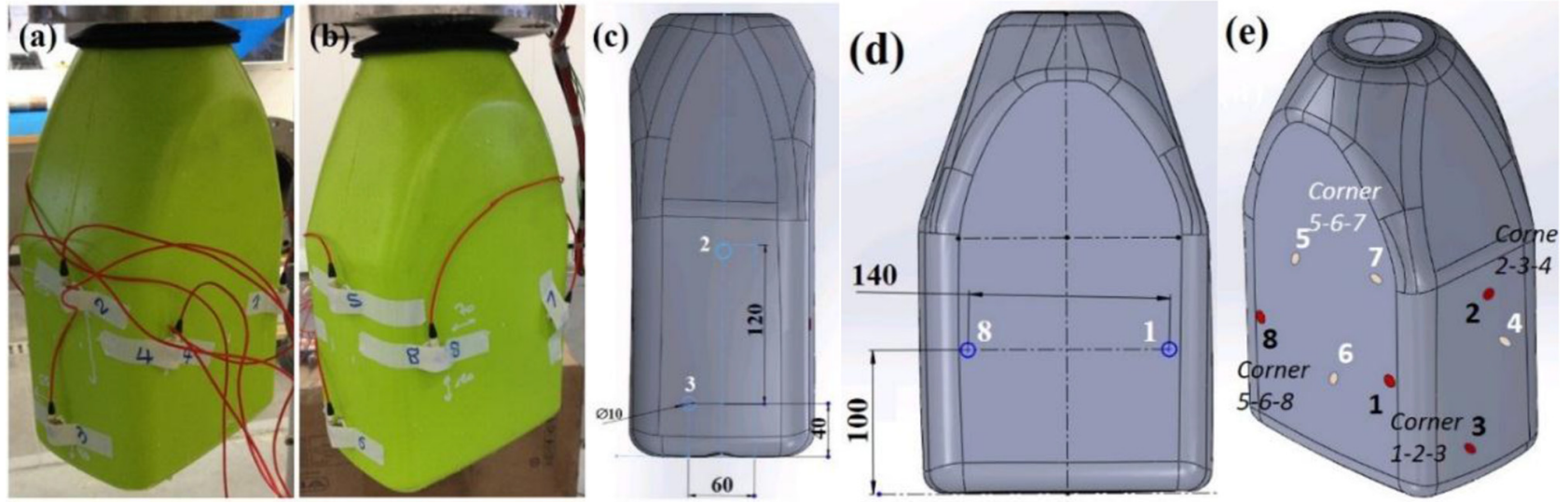

Fig. 21. Positioning the sensors in a «triangle».

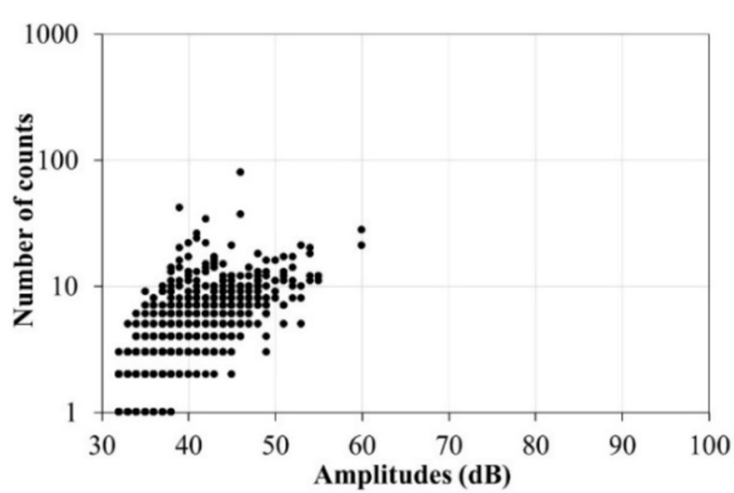

(a)

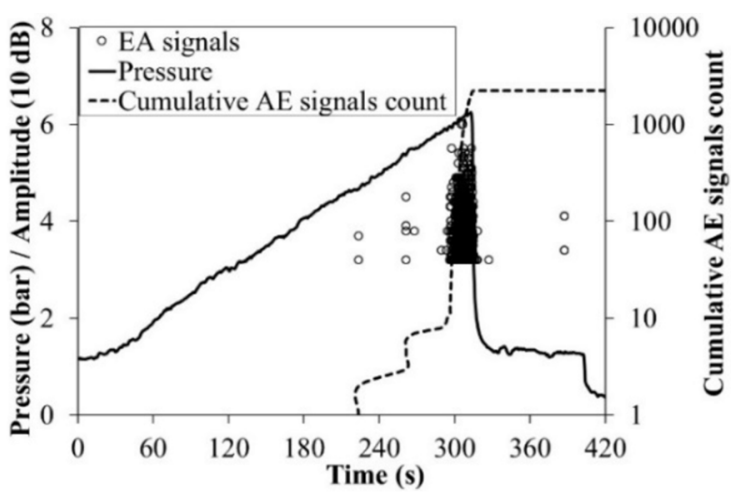

(b)

Fig. 22. Test 3. (a) Number of counts vs amplitude (dB). (b) Monotonic test under internal pressure - internal pressure, amplitude vs time and cumulative AE signals count vs time.

visual observation of the inside of the bottle leads us to notice that two cracks on the bottom of the bottle (at the level of the edges) and one on a vertical edge appeared. Contrary to the previous bottle, the rupture zones here are really located at the edges, i.e. in the angle (Fig. 24).

It is not easy to associate the AE signals detected by sensors 1, 2 and 3 with damaged areas. However, depending on their initial positions on the bottle, the number of costs captured by the sensors and the rupture zones observed, it is possible to perform the following analyses:

- Sensor 1 mainly detected the crack along the vertical edge noted $\mathbf{A}$ with amplitude up to $55 \mathrm{~dB}$ and secondary damage to the large horizontal edge.

- Sensor 2, which captured the least cost than sensors 1 and 3 , appears to have perceived acoustic activity from the small horizontal 2 and/or vertical edge $\mathbf{A}$ and $\mathbf{B}$. It is difficult to make a decision here, as this sensor is almost at an iso-distance from both edges.

- Sensor 3, which is also located at almost the same distance between the damaged edges.

The maximum amplitudes measured by the sensors are in accordance with their positions. Thus, the maximum values are approximately 55, 40 and $45 \mathrm{~dB}$ respectively for sensors 1, 2 and 3 . This obviously indicates the importance of the positioning of the sensors in relation to the location of the damage. On the other vertical edges no damage is observed, which may explain why the other sensors $(4,5,7,8)$ did not detect any signals. In view of the damage areas, it is reasonable to assume that, although the signals are weak, sensors 3 and 6 (Fig. 24b) have detected damage to the horizontal edges $\mathbf{B}$ and $\mathbf{C}$ of the bottom of the bottle (Fig. 23). Since it is likely that the signals perceived by sensor 1 correspond to the damage to the vertical edge but also to the two horizontal edges of the bottom of the bottle. Although the position of the sensors has been changed, it is difficult to localize the rupture zones perfectly. Despite it is a different polyolefin, one finds a very similar scenario, the observations with Ray-X are identical (Fig. 24a) and the AE signals present the same characteristics with the distributions A-FRQ function of RA value similar (Fig. 24b).

\section{Discussion}

In the first test on structure, it was shown that with the very first hits, it was possible to detect the first damage originating at the level of the initial defects in the bottle. 

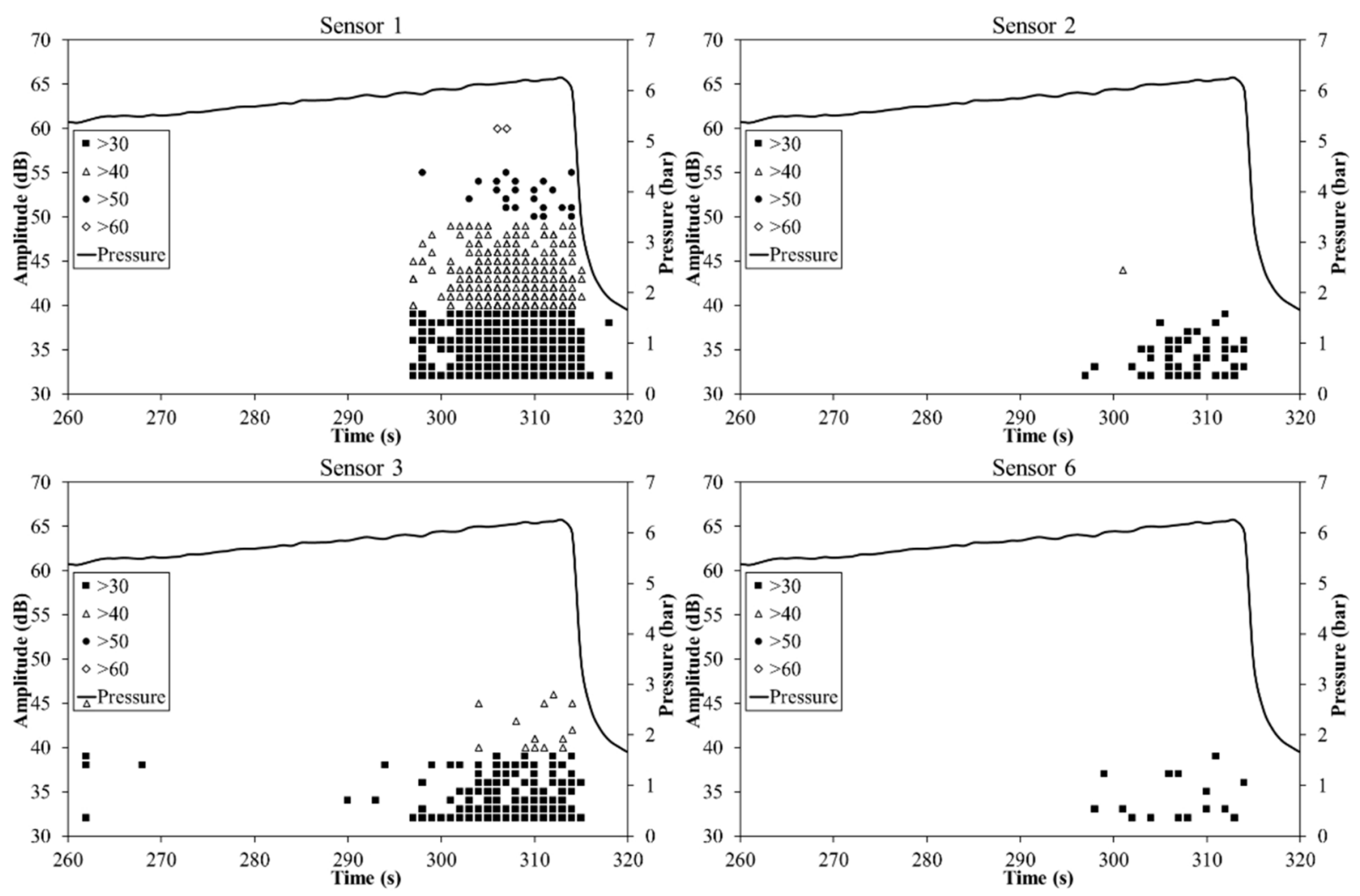

Fig. 23. Amplitude of signals on different sensors.

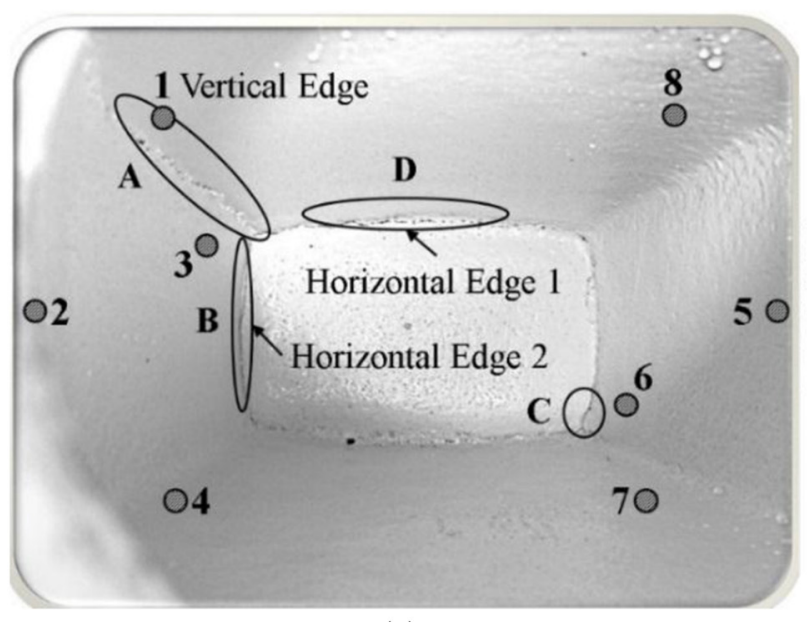

(a)

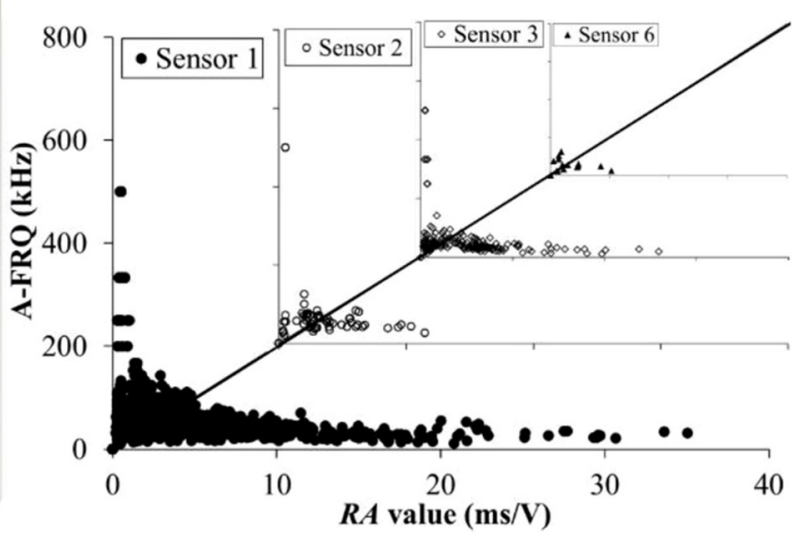

(b)

Fig. 24. (a) Photo of the inside of the bottle after testing and disassembly areas of breakage of the inner skin of the bottle. (b) Relationship between average frequency (A-FRQ) and RA value.

Indeed, the thin thickness of the inner skin, subjected to internal pressure, generates in these localized areas a significant deformation gradient which should be conducive to cavitation. Once areas weakened by damage open, it seems that the inner skin is under the effect of pressure and therefore localized swelling tears. After these localisation propagates in the structure. For this test, where the objective was to interrupt the test as soon as the first hits appeared, it appears that the chosen classification is completely consistent. Thus, the high A-FRQ and low RA values are similar to those observed in the tensile test.

The second test on structure was intended to go a little further in the fracture without a total fracture of the structure. The results showed that there was a match between the hits detected by the sensors, the position of the sensors, the position of the cracks and their lengths. 

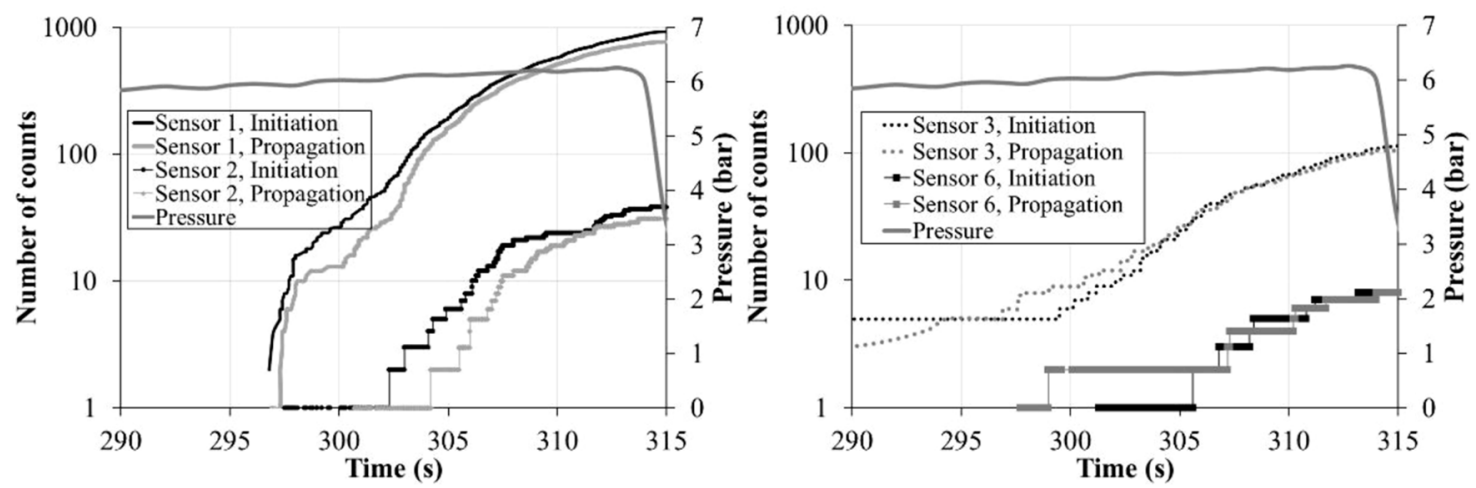

Fig. 25. Relation between load and crack generation on the time domain - Test 3 .

Table 2. Results of the crack classification (\%) - Test 1.

\begin{tabular}{llll}
\hline Sensor & Number of hits & Initiation & Tearing \\
\hline 1 & 16 & 100.0 & 0.0 \\
3 & 4 & 100.0 & 0.0 \\
\hline
\end{tabular}

Table 3. Results of the crack classification (\%) - Test 2 .

\begin{tabular}{llll}
\hline Sensor & Number of hits & Initiation & Tearing \\
\hline 1 & 12 & 50.0 & 50.0 \\
2 & 23 & 82.6 & 17.4 \\
3 & 2 & 0.0 & 100.0 \\
4 & 88 & 52.3 & 47.7 \\
5 & 7 & 42.9 & 57.1 \\
7 & 3 & 100.0 & 0.0 \\
\hline
\end{tabular}

In test 3 , one can imagine that the initiation of the damage originates in the angle at the intersection of the three damaged edges. Sensor 1 has the most AE hits (1703). The sensor is farthest from the damage areas. As it is not possible to know where the hits come from compared to the breakthrough propagations; it should be then looked at these results as a whole. Thus, for sensors 1, 2 and 3, the average is about $50 \%$ for initiation and $50 \%$ for tearing. For sensor 6 , which is the only one to have picked up acoustic activity due to the start of damage in the opposite angle to the three sensors 1,2 and 3 , the proportions between initiation and tearing are in adequacy between what was observed previously.

Tables 2-4 quantify the number of hits associated with each phenomenon (initiation-tearing) separated by the slope line 20 that has been chosen. In the case that the proportion of the RA value and the average frequency is set to 1:20 as given for test 1 (Tab. 2) a total of signals related to damage initiation are observed as for the tensile test. For test 2 (Tab. 3), sensors 2 and 7 detected a majority of signals related to damage initiation, the ratio of initiation becomes $82.6 \%$ and $100 \%$ respectively and $17.4 \%$ and $0 \% \mathrm{AE}$ hits are classified as tearing. On the
Table 4. Results of the crack classification (\%) - Test 3 .

\begin{tabular}{llll}
\hline Sensor & Number of hits & Initiation & Tearing \\
\hline 1 & 1703 & 54.7 & 45.3 \\
2 & 74 & 55.1 & 44.9 \\
3 & 230 & 52.0 & 48.0 \\
6 & 18 & 50.0 & 50.0 \\
\hline
\end{tabular}

other hand, sensors 1, 4 and 5 measured a distribution of about $50 \%$. Finally, in test 3 (Tab. 4), for all sensors, there is a perfect distribution of $\mathrm{AE}$ hits of about $50 \%$ for initiation and tearing. Some cases are $100 \%$ but for a very small number of hits on the corresponding sensor. Test 2 was interrupted after $135 \mathrm{AE}$ hits, 111 of which were captured by sensors 2 and 4, placed at the bottom of the bottle (Tab. 3). According to test 1, the damage is initiated approximately at the mid-point of the bottle. Although sensors 1, 3,5 and 7 are closer to these areas, sensors 2 and 4 have the most hits.

The correlation of the other AE parameters (e.g. energy, duration time as a function of amplitude) did not allow the separation of distinct families of damage mechanisms. In addition, tomographic observations show cavitation and tearing in the case of the bottle. The two parameters used (RA value and A-FRQ) for other materials that are very different from the point of view of their microstructure (concrete) made it possible to understand the sequence of the initiation of small damages and the propagation of tears.

From the point of view of the kinetics, Figure 25 gives the temporal evolutions of the numbers of counts, relative to the two mechanisms during the test, a perfect synchronization of the signals is observed with the curves of the phenomenon of initiation in advance of phase of that of spread, this point correspond to evolution in the structure of process zone.

Figure 26 shows the AE event count $(N)$ and amplitude $\left(A_{\mathrm{mv}}\right)$ obtained from the bottle during the static internal pressure test. The data at $N \geq 1$ was plotted, since a few $\mathrm{AE}$ signals were generated due to the swelling process. From equation (8), the value of the coefficient $m$ can be identified directly from the points in Figure 26. 


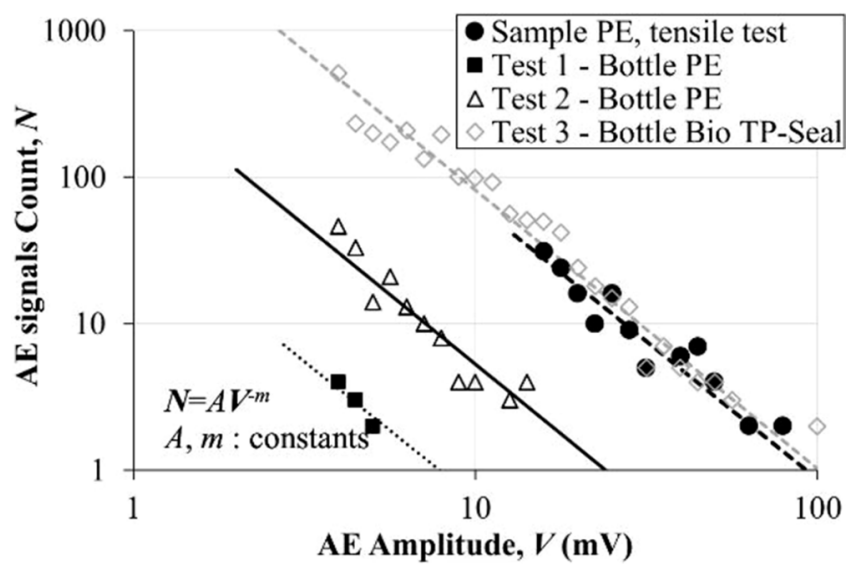

Fig. 26. Relationship between the number of AE signals and the AE amplitude obtained from tensile test and bottle tests.

For the tensile test, the value of $m$ is 1.90 for amplitudes greater than $42 \mathrm{~dB}(12.6 \mathrm{~V})$. For tests 1, 2 and 3 on bottles, where there was sufficient acoustic activity to exploit these quantities, the value of $m$ is $1.90,1.90$ and 1.88 , respectively. Fracture behaviour is correlated with this slope, consequently the value obtained for this semicrystalline material is representative of crack growth on energy that is between steel and rubber. For fatigue crack growth of a steel material, it is reported that $m \approx 2$ [41]. Yamabe et al. [40] determined that $m=1.8$ from the rubber material for the static crack growth test.

\section{Conclusions}

The tests were carried out on tensile test specimens and bottles. The effect of the size of the structure and the effect of the propagation medium on the generated waves and recorded descriptors are not taken into account. The number of sensors was not sufficient to perform such an analysis. However, the comparison of the structural test records with those of the tensile test is relevant. The distance between the sensors and the acoustic signals is in areas of similar dimensions. In addition, due to the very high damping of the polymer material, the sensors can only record signals located nearby and therefore equivalent to those of the tensile test. The scenario of rupture of the internal skin of the sandwich structure (bottle) subjected to an internal water pressure is proposed on the basis of a crossing of $\mathrm{AE}$ measurements and radiological observations. Three tests on this bottle with polymer skins such as $\mathrm{PE}$ show that the use of AE can capture structural damage and/or failures. These results are concretely complementary to the work of the literature [28-32]. The passage from a tensile specimen to an industrial sandwich structure (rotomoulded bottle) is therefore validated. In a complex environment (water under pressure), with a PE sandwich material, it is possible to detect the first stages of damage at 6 bar and the propagation of cracks up to the high breaking stage of the inner skin with acoustic emission. The study of acoustic signals in association with X-ray observations correlated the RA and A-FRQ parameters with the mechanisms. Although the waveforms were not saved during the tests, the use of $\mathrm{AE}$ parameters such as energy and duration time did not provide additional information to classify initiation and tearing. In addition, the damping of the material and structure makes their analysis very difficult. At this stage, no proposals have been made in the literature for polymers and even less for polymer sandwich structures. This work initiates a new approach that will have to be confirmed in the future by additional tests (shear, crack growth, etc.) in order to complete the understanding of the evolution of the damage.

\section{Nomenclature}

$\begin{array}{ll}\varepsilon_{l} & \text { Logarithmic longitudinal strain }(\%) \\ \varepsilon_{t} & \text { Logarithmic transverse strain }(\%) \\ \sigma_{v} & \text { True stress (or Cauchy stress) }(\mathrm{MPa}) \\ \tau_{d} & \text { Duration time } \tau_{d}(\mathrm{~ms}) \\ \tau_{\mathrm{r}} & \text { Rise time }(\mathrm{ms}) \\ A_{\mathrm{mdB}} & \text { Maximum amplitude }(\mathrm{dB}) \\ A_{\mathrm{mv}} & \text { Maximum amplitude (Volts) } \\ \mathrm{RA} & \text { RA value }(\mathrm{ms} / \mathrm{V}) \\ \mathrm{A}-\mathrm{FRQ} & \text { Average frequency }(\mathrm{kHz}) \\ \mathrm{AE} C & \text { AE count } \\ G & \text { Preamplifier gain }(\mathrm{dB}) \\ N & \text { AE signal count } \\ S_{0} & \text { Initial cross-section area }\left(\mathrm{mm}^{2}\right) \\ S & \text { Actual cross-section area }\left(\mathrm{mm}^{2}\right) \\ F & \text { Axial load (N) }\end{array}$

Acknowledgments. This work was funded by the French Government program "Investissements d'Avenir" (EQUIPEX GAP, reference ANR-11-EQPX-0018). The authors would like to thank Total Petrochemicals for their financial support for this research. The authors would like to thank Total Refining \& Chemicals for the provision of structures (bottles).

\section{References}

[1] M. Emami, E. Takacs, J. Vlachopoulos, Study of foaming mechanisms in rotational molding, ANTEC, 2010

[2] M. Emami, M.R. Thompson, E. Takacs, J. Vlachopoulos, Rheological effects on foam processing in rotational molding, ANTEC, 2011

[3] M. Emami, M.R. Thompson, E. Takacs, J. Vlachopoulos, Rheological aspects of rotational foam molding, PPS, 2011

[4] M. Emami, E. Takacs, M.R. Thompson, J. Vlachopoulos, E. Maziers, Visual studies of model foam development for rotational molding processes, Adv. Poly. Technol. 32, 809-821 (2013)

[5] E. Maziers, Skin-foam-skin TP-Seal ${ }^{\circledR}$ rotomolded structures: a new concept for the production of car bodies for urban mobility, Plastics Eng. 69, 40-42 (2013)

[6] J.E. Arbaoui, Etude comparative et caractérisations mécaniques des structures sandwichs multicouches, Thèse, Université Paul Verlaine Metz, 2009

[7] A. Mirzapour, M.H. Beheshty, M. Vafayan, The response of sandwich panels with rigid polyurethane foam cores under flexural loading, Iran. Polym. J. 14, 20182-20188 (2005) 
[8] F.P. Yang, Q.Y. Lin, J.J. Jiang, Experimental study on fatigue failure and damage sandwich structure with PMI foam core, Fatigue Fract. Eng. Mater. Struct. 38, 456-465 (2015)

[9] K.S. Narayanaswamy, H.N. Vidyasagar, N. Ranapratap Reddy, Bending characteristics of open cell polymer foam sandwich structure, Procedia Mater. Sci. 5, 1906-1914 (2014)

[10] A. Cernescu, J. Romanoff, Bending deflection of sandwich beams considering local effect of concentrated force, Compos. Struct. 134, 169-175 (2015)

[11] L.S. Sutherland, S.C. Guedes, Impact characterisation of low fibre-volume glass reinforced polyester circular laminated plates, Int. J. Impact Eng. 31, 1-23 (2005)

[12] J.P. Dear, H. Lee, S.A. Brown, Impact damage processes in composite sheet and sandwich honeycomb materials, Int. J. Impact Eng. 32, 130-154 (2005)

[13] R. Vignjevic, M. Meo, G. Marengo, The response of honeycomb sandwich panels under low-velocity impact loading, Int. J. Mech. Sci. 47, 1301-1325 (2005)

[14] H.Y. Choua, A.P. Mouritz, M.K. Bannister, A.R. Bunsell, Acoustic emission analysis of composite pressure vessels under constant and cyclic pressure, Compos. Part A Appl. Sci. Manuf. 70, 111-120 (2015)

[15] J.R. Kwon, G.J. Lyu, T.H. Lee, J.Y. Kim, Acoustic emission testing of repaired storage tank, Int. J. Press. Vessel Pipping 78, 373-378 (2001)

[16] M. Burman, M. Battley, Acoustic emission monitoring of foam core sandwich composites, J Sandwich Struct. Mater. 1, 147-175 (1999)

[17] I. Ben Ammar, C. Karra, A. El Mahi, R. El Guerjouma, M. Haddar, Mechanical behaviour and acoustic emission technique for detecting, Appl. Acoust. 86, 106-117 (2014)

[18] C. G'sell, J.J. Jonas, Yield and transient effects during the plastic deformation of solid polymers, J. Mater. Sci. 16, 1956-1974 (1981)

[19] A. Galeski, Strength and toughness of crystalline polymer systems, Prog. Polym. Sci. 28, 1643-1699 (2003)

[20] A. Pawlak, A. Galeski, A. Rozanski, Cavitation during deformation of semi-crystalline polymers, Prog. Poly. Sci. 39, 921-958 (2014)

[21] S. Castagnet, Y. Deburck, Relative influence of microstructure and macroscopic triaxiality on cavitation damage in a semi-crystalline polymer, Mater. Sci. Eng. A Struct. Mater. 448, 56-66 (2007)

[22] C. G'sell, A. Dahoun, Evolution of microstructure in semicrystalline polymers under large plastic deformation, Mater. Sci. Eng. 175, 183-199 (1994)

[23] L. Lin, A.S. Argon, Structure and plastic deformation of polyethylene, J. Mater. Sci. 29, 294-323 (1994)

[24] C.J.G. Plummer, A. Goldberg, A. Ghanem, Micromechanisms of slow crack growth in polyethylene under constant tensile loading, Polymer 42, 9551-9564 (2001)
[25] H.B.H. Hamouda, M. Simoes-Betbeder, F. Grillon, P. Blouet, N. Billon, Creep damage mechanisms in polyethylene gas pipes, Polymer 42, 5425-5437 (2001)

[26] D. Betteridge, P.A. Connors, T. Lilley, N.R. Shoko, M.E.A. Cudby, D.G.M. Wood, Analysis of acoustic emissions from polymers, Polymer 24, 1206-1212 (1983)

[27] F. Ronkay, T. Czigany, Cavity formation and stressoscillation during the tensile test of injection molded specimens made of PET, Polym. Bull. 57, 989-998 (2006)

[28] J. Bohse, Acoustic emission characteristics of micro-failure processes in polymer blends and composites, Compos. Sci. Technol. 60, 1213-1226 (2000)

[29] R. Qian, T. Wang, J. Shen, Acoustic emission during stretching of polymers, Polym. Commun. 2, 168-175 (1983)

[30] A. Galeski, E. Piorkowska, L. Koenczoel, E. Baer, Acoustic emission during polymer crystallization, J. Polym. Sci. Part B Polym. Phys. 28, 1171-1186 (1990)

[31] E.T. Teofilo, M.S. Rabello, The use of acoustic emission technique in the failure analysis of PET, Polym. Test. 45 68-75 (2005)

[32] N. Casiez, S. Deschanel, T. Monnier, O. Lame, Acoustic emission from the initiation of plastic deformation of polyethylenes during tensile tests, Polymer 55, 6561-6568 (2014)

[33] F.P.C. Gomes, A. Bovell, G.P. Balamurugan, M.R. Thompson, K.G. Dunn, Evaluating the influence of contacting fluids on polyethylene using acoustic emissions analysis, Polym. Test. 39, 61-69 (2014)

[34] A. Nielsen, Acoustic emission source based on pencil lead breaking, The Danish Welding Institute Publication 80, 15-20 (1980)

[35] K. Jemielniak, Some aspects of acoustic emission signal preprocessing, J. Mater. Proc. Tech. 109, 242-247 (2001)

[36] A. Malpot, Etude du comportement en fatigue d'un composite à matrice polyamide renforcé d'un tissue de fibres de verre pour application automobile, Thèse, ENSMA, 2017

[37] JC MS-III B5706, Monitoring method for active cracks in concrete by acoustic emission, Japan: Federation of Construction Materials Industries, 2003

[38] K. Ohno, M. Ohtsu, Crack classification in concrete based on acousyic emission, Const. Buld. Mater. 24, 2339-2346 (2010)

[39] M. Ohtsu, The history and development of acoustic emission in concrete engineering, Mag. Concr. Res. 48, 321-330 (1996)

[40] J. Yamabe, T. Matsumoto, S. Nishimura, Application of acoustic emission method to detection of internal fracture of sealing rubber material by high-pressure hydrogen decompression, Polym. Test. 30, 76-85 (2011)

[41] S. Yuyama, T. Kishi, Y. Hisamatsu, Effect of environmental, mechanical conditions, and materials characteristic on $\mathrm{AE}$ behaviour during corrosion fatigue processes of an austenitic stainless steel, Nucl. Eng. Des. 81, 345-355 (1984)

[42] A. Marquez-Lucero, C. G'sell, K.W. Neale, Experimental investigation of neck propagation in polymers, Polymer $\mathbf{3 0}$, 636-642 (1989)

Cite this article as: E. Lainé, J.-C. Grandidier, M. Cruz, A.-L. Gorge, C. Bouvy, G. Vaes, Acoustic emission description from a damage and failure scenario of rotomoulded polyolefin sandwich structure subjected to internal pressure for storage applications, Mechanics \& Industry 21, 105 (2020) 